\title{
Three-dimensional visualization of ensemble weather forecasts - Part 1: The visualization tool Met.3D (version 1.0)
}

\author{
M. Rautenhaus ${ }^{1}$, M. Kern ${ }^{1}$, A. Schäfler ${ }^{2}$, and R. Westermann ${ }^{1}$ \\ ${ }^{1}$ Computer Graphics \& Visualization Group, Technische Universität München, Garching, Germany \\ ${ }^{2}$ Deutsches Zentrum für Luft- und Raumfahrt, Institut für Physik der Atmosphäre, Oberpfaffenhofen, Germany \\ Correspondence to: M. Rautenhaus (marc.rautenhaus@tum.de)
}

Received: 4 February 2015 - Published in Geosci. Model Dev. Discuss.: 27 February 2015

Revised: 23 June 2015 - Accepted: 25 June 2015 - Published: 31 July 2015

\begin{abstract}
We present "Met.3D", a new open-source tool for the interactive three-dimensional (3-D) visualization of numerical ensemble weather predictions. The tool has been developed to support weather forecasting during aircraft-based atmospheric field campaigns; however, it is applicable to further forecasting, research and teaching activities. Our work approaches challenging topics related to the visual analysis of numerical atmospheric model output - 3-D visualization, ensemble visualization and how both can be used in a meaningful way suited to weather forecasting. Met.3D builds a bridge from proven 2-D visualization methods commonly used in meteorology to 3-D visualization by combining both visualization types in a 3-D context. We address the issue of spatial perception in the 3-D view and present approaches to using the ensemble to allow the user to assess forecast uncertainty. Interactivity is key to our approach. Met.3D uses modern graphics technology to achieve interactive visualization on standard consumer hardware. The tool supports forecast data from the European Centre for Medium Range Weather Forecasts (ECMWF) and can operate directly on ECMWF hybrid sigma-pressure level grids. We describe the employed visualization algorithms, and analyse the impact of the ECMWF grid topology on computing 3-D ensemble statistical quantities. Our techniques are demonstrated with examples from the T-NAWDEX-Falcon 2012 (THORPEX North Atlantic Waveguide and Downstream Impact Experiment) campaign.
\end{abstract}

\section{Introduction}

Weather forecasting requires meteorologists to explore large amounts of numerical weather prediction (NWP) data, and to assess the uncertainty of the predictions. Visualization methods that facilitate fast and intuitive exploration of the data hence are of particular importance. In practice, the forecasting process for the most part relies on two-dimensional (2-D) visualization methods. Meteorologists use weather maps, vertical cross sections and a multitude of meteorological diagrams to depict the data. From these image sources, they build "mental models" of the three-dimensional (3-D), timevarying forecast atmosphere inside their heads (Hoffman and Coffey, 2004; Trafton and Hoffman, 2007).

Despite the 3-D nature of the atmosphere, 3-D visualization methods have not found widespread usage, even though there have been promising attempts in the 1990s and early 2000s that suggested added value (Treinish and Rothfusz, 1997; Koppert et al., 1998; McCaslin et al., 2000). Various hindering factors are discussed in the literature, including resistance of forecasters to adapt to new 3-D visualization methods that are decoupled from their "familiar" 2-D products (Koppert et al., 1998; Szoke et al., 2003), problems with spatial perception in 3-D renderings (Szoke et al., 2003), as well as issues due to limited performance (Treinish and Rothfusz, 1997) and the need for dedicated graphics workstation hardware (Koppert et al., 1998).

In addition to 3-D space and time, forecast visualization has in recent years become more challenging through the increased use of ensemble weather predictions - sets of forecast runs whose distribution provides information on forecast uncertainty (e.g. Gneiting and Raftery, 2005; Leutbecher and Palmer, 2008). The development of visualization meth- 
ods that depict the uncertainty derived from ensemble data is an active topic of research not only for weather forecast ensembles (Obermaier and Joy, 2014). Yet again, ensemble visualization techniques related to weather forecasting published so far mainly focus on two dimensions as well (e.g. Potter et al., 2009; Sanyal et al., 2010).

In this paper we introduce a new open-source visualization tool, "Met.3D", that provides interactive 3-D visualization techniques for ensemble prediction data. There has been an immense progress in mainstream graphics hardware capabilities in recent years. Making use of these developments, Met.3D facilitates interactive visualization of presentday NWP data sets on consumer hardware. The tool has been developed as a new effort to demonstrate the feasibility of using 3-D visualization for forecasting, this time also considering uncertainty information from ensemble data sets. It is intended to be used for actual forecasting tasks, as well as a platform to implement and evaluate new 3-D and ensemble visualization techniques.

The work presented in this paper has been inspired by a particular application, forecasting the weather situation to plan research flights during aircraft-based field campaigns. We focus on this application throughout the paper at hand. However, Met.3D is applicable to a broader range of forecasting and visual data analysis tasks. Both fast exploration and uncertainty assessment play a major role in campaign forecasting:

1. When investigating suitable meteorological conditions to specify the route of a research flight (that is, waypoints in 3-D space and time), the forecaster is required to quickly identify atmospheric features relevant to the flight and to communicate findings to colleagues. Upper-level features typically important to flights with high-flying aircraft are of an inherently 3-D nature (for example, clouds, jet streams or the tropopause). From our experience in campaigns with DLR (German Aerospace Centre) involvement, visualization used during campaigns has been solely based on 2-D methods, typically with limited interactivity. We are hence interested in investigating how 3-D visualization methods and interactivity (to quickly navigate the data space) can be used to aid the exploration.

2. Assessing the forecast's uncertainty has become indispensable as flights frequently have to be planned multiple days before take off (typically 3-7 days; the medium forecast range) to obtain the required approval from air traffic authorities. While the use of ensemble predictions has been reported for recent field campaigns (e.g. Wulfmeyer et al., 2008; Elsberry and Harr, 2008; Ducrocq et al., 2014; Vaughan et al., 2015), they have, to the best of our knowledge, not been used to create specific interactive forecast products for flight planning. However, ensembles provide valuable information; for example, 3-D probability fields for the occurrence of a targeted atmospheric process or feature can be derived. Potential flight routes can be planned in regions in which the probability is high. An open question, however, is how can the ensemble data be visualized to improve flight planning in the medium forecast range.

Our objective is to use interactive 3-D visualization of ensemble predictions from the European Centre for Medium Range Weather Forecasts (ECMWF) to improve the forecast process for field campaigns. The work has been stimulated by the forecast requirements of a specific field campaign, the international T-NAWDEX-Falcon campaign (THORPEX North Atlantic Waveguide and Downstream Impact Experiment - Falcon, hereafter TNF). TNF took place in October 2012 with the objective to take in situ measurements in warm conveyor belts (WCBs), airstreams in extratropical cyclones that lift warm and moist air from near the surface to the upper troposphere (e.g. Browning and Roberts, 1994). Schäfler et al. (2014) provided details on the campaign and its flight planning. The major forecasting challenge was to predict the likelihood of WCB occurrence within aircraft range. This was expressed by a number of forecast questions that guided the development of Met.3D:

A. How will the large-scale weather situation develop over the next week, and will conditions occur that favour WCB formation?

B. How uncertain are the weather predictions?

C. Where and when, in the medium forecast range and within the spatial range of the aircraft, is a WCB most likely to occur?

D. How meaningful is the forecast of WCB occurrence?

E. Where will the WCB be located relative to cyclonic and dynamic features?

In a recent ECMWF newsletter article (Rautenhaus et al., 2014), we provided a brief overview of our work. It is the purpose of this publication to describe the techniques we have developed in detail and to present our solutions to particular challenges.

We split our work into two parts, structured as follows. In this paper, we introduce Met.3D. We discuss challenges related to interactive 3-D visualization and present techniques that address questions $\mathrm{A}$ and $\mathrm{B}$.

To put our work in the context of the literature, we review recent work in meteorological and ensemble visualization in Sect. 2. Section 3 presents Met.3D's visualization capabilities. When introducing 3-D visualization to forecasting, we need to consider that the 2-D visualization methods commonly used in meteorology provide many advantages (for example, spatial perception) and that meteorologists are used to working with them. In a 3-D forecast tool to be used in practice, we hence have to be careful not to replace proven 
2-D methods, but to put them into a 3-D context and to use 3 -D visualization to add value. We address the challenges of creating such a "bridge" from 2-D to 3-D visualizations, of improving spatial perception of 3-D renderings and of designing interactive methods that provide fast and easy visual access to ensemble information. A supplementary video containing real-time screen recordings of examples shown in Sect. 3 demonstrates the performance of Met.3D on midrange consumer hardware.

To avoid time-consuming pre-processing of the forecast data prior to visualization, Met.3D operates directly on the ECMWF hybrid sigma-pressure model grid. The characteristics of the data and resulting challenges for visualization are discussed along with Met.3D's visualization algorithms and system architecture in Sect. 4. Section 5 discusses the efficient yet accurate computation of statistical quantities from the ensemble predictions. When computing statistical quantities on a per grid-point basis an error is introduced, since the vertical positions of the ECMWF model grid points vary between members. Regridding to a common grid is a solution, albeit time-consuming and hence undesirable for real-time visualization. We analyse the error introduced when ignoring such a regridding and provide advice on how to handle the issue. Section 6 provides information on code availability, before the paper is concluded in Sect. 6 .

In the second part of this study (Rautenhaus et al., 2015, hereafter "Part 2"), we address forecast questions $\mathrm{C}$ to E. A method to compute 3-D WCB probabilities from Lagrangian particle trajectories is introduced and evaluated, and Met.3D is extended by a technique to visually analyse the derived probabilities. To demonstrate the added value of 3-D visualization for forecasting, we present a comprehensive case study with detailed meteorological interpretations of a forecast case of TNF. The case study uses methods from both papers and illustrates how Met.3D can be used in practice. Readers primarily interested in the application of Met.3D should read Sect. 3 in this part, skip the technical sections and proceed to the case study in Part 2.

\section{3-D and ensemble visualization in meteorology}

Our work is related to 3-D visualization in meteorology and to uncertainty and ensemble visualization.

\section{$2.13-D$ visualization in meteorology}

Visualization tools in meteorology can be distinguished with respect to application in a research setting and application in an operational forecast setting (Papathomas et al., 1988). As Koppert et al. (1998) point out, a tool in an operational setting should offer techniques tailored to the specific forecasting task and not confuse the forecaster with large sets of parameters that need to be configured. A research setting, on the other hand, demands a tool that is flexible to adapt to dif- ferent exploration tasks and data formats. Its visualizations should be highly configurable by the user.

In forecasting, 2-D visualization systems prevail. With respect to field campaigns with DLR involvement, the Mission Support System (MSS) is frequently used, a tool that generates horizontal and vertical 2-D sections of the forecast data upon user request (Rautenhaus et al., 2012). This tool motivated the design of our proposed bridge from 2-D to 3-D that we describe in Sect. 3. Further 2-D systems that have been applied include the German Weather Service (DWD) NinJo workstation (Heizenrieder and Haucke, 2009) and the ECMWF Metview software (Russell et al., 2010).

The few reports on the usage of 3-D visualization of atmospheric model data in forecasting date to the 1990s and early 2000s. Treinish (1996), Treinish and Rothfusz (1997) and Treinish (1998) reported on experiments with 3-D visualization for local forecasting during the 1996 Olympic Games in Atlanta. They concluded that an advantage of their 3-D methods was "that they virtually eliminated the need to laboriously evaluate numerous two-dimensional images", however, noted a lack of interactivity due to limitations in computational performance. Schröder (1997), Lux and Frühauf (1998) and Koppert et al. (1998) presented "RASSIN" and its successor "VISUAL", a 3-D forecasting system for usage within the DWD. Discussing their experience with an operational test of the software, Koppert et al. (1998), too, point out the importance of system performance for user acceptance. They furthermore highlight the need for common concepts of operations (user interface and workflow) when forecasters are asked to transition from a 2-D to a 3-D environment.

McCaslin et al. (2000) presented "D3D” (Display 3D), a 3-D software built at the United States Forecast Systems Laboratory (FSL) on top of the "Vis5D" tool (Hibbard and Santek, 1990). D3D's user interface was designed to match that of the 2-D "D2D" (Display 2D) software in use at the National Weather Service Weather Forecast Offices (WFOs). "Real-time forecast exercises" were conducted to evaluate the value of 3-D visualization, and the software was installed at a number of WFOs. A few case studies were presented, including usage of D3D for the examination of tropical cyclones (Watson et al., 2002), the usage of 3-D trajectories (Barjenbruch et al., 2002), and the analysis of the synoptic situation during a tornado outbreak (Nietfeld, 2003). Szoke et al. (2003) reported on experiences gained with the system. They not only discuss the reluctance of forecasters to switch from 2-D to 3-D, but also confidently state that for forecasters trained with D3D it is "hard to deny that examining the atmosphere using a 3-D tool is not more effective and complete than using 2-D displays". Szoke et al. (2003) also positively reported on the interactivity introduced by their system. Interactively moveable vertical soundings and cross sections, for example, were very well perceived by the forecasters. There was also an approach to ensemble visualization with D3D. Alpert (2003) suggest to interpret the ensemble 
dimension as the vertical coordinate in Vis5D and to view a 2-D map of an ensemble product as a 3-D isosurface. Subsequently, Nietfeld (2006) reported on the application of 3-D techniques in a WFO to visualize observed radar data in the forecast process, using the "GR2Analyst" software.

With respect to research environments, 3-D visualization is more frequently used. Early approaches in the 1970s and 1980s used mainframe computers to create 3-D views or animations of atmospheric observations and numerical model output (e.g. Grotjahn and Chervin, 1984; Hibbard, 1986; Papathomas et al., 1988; Hibbard et al., 1989; Schiavone and Papathomas, 1990, and references therein). For example, Wilhelmson et al. (1990) created an award-winning (cf. Middleton et al., 2005) animation movie of a numerically modelled storm, a project that at that time still required multiple months and a large amount of computer time (Wilhelmson et al., 1990). Since around 1990, a number of workstation and desktop visualization tools have appeared. Vis5D, mentioned above, became a major 3-D visualization tool in meteorology and was widely used into the 2000s (Hibbard, 2005; Middleton et al., 2005). However, its development was discontinued. A number of other, mostly general-purpose, systems that have been used in the atmospheric sciences are listed by Schröder (1997), Böttinger et al. (1998) and Middleton et al. (2005). They include the commercial systems "Application Visualization System" (Upson et al., 1989; Favre and Valle, 2005), "Iris Explorer" (Walton, 2005), the "IBM Data Explorer" (Abram and Treinish, 1995; Watson, 1995, later renamed to "OpenDX" and made open source; discontinued in 2007), and "amira" (Stalling et al., 2005; now "Avizo").

More recently, prominent tools include "Vapor" (Norton and Clyne, 2012; Clyne et al., 2007) and the Unidata "Integrated Data Viewer" (IDV) (Murray and McWhirter, 2007; Murray et al., 2009). Vapor is an open-source 3-D visualization software developed at the United States National Centre for Atmospheric Research. It features a number of 3-D visualization techniques to view time-varying gridded data sets; however, it does not provide techniques for ensemble data or forecasting functionality. IDV is a comprehensive Java application for the analysis and visualization of geosciences data. It is based on the "Visualization for Algorithm Development" (VisAD) library (e.g. Hibbard, 1998, 2005) and supports a variety of visualization methods, including some 3-D support. For example, Yalda et al. (2012) use IDV's 3-D capabilities for interactive immersion learning. On a broader scope, "Paraview" (Henderson et al., 2004) is an open-source, general-purpose visualization tool that can also be used with meteorological data. In the context of a graduate university course, Dyer and Amburn (2010) investigated how Paraview can be used in a meteorological setting. Also, commercial general-purpose systems with 3-D capabilities that are frequently used in the atmospheric domain include "Interactive Data Language" (IDL) (e.g., cf. Middleton et al., 2005) and "Avizo Green" (e.g. Böttinger et al., 2013).
3-D visualization has also been used for virtual reality applications in teaching (e.g. Gallus et al., 2003, 2005).

A major reason why 2-D methods are often preferred in the atmospheric sciences is that they are well suited to convey quantitative information, as Middleton et al. (2005) point out in a survey of visualization in meteorology. 2-D contour lines and colour mappings can be used to convey a large range of data values. In a 3-D depiction, only a small number of isosurfaces can be displayed without cluttering and occlusion. However, a 3-D image is able to convey spatial structure in all three dimensions, a distinct advantage compared to 2-D methods. On the downside, spatial perception is more challenging in 3-D. Determining the location of a data feature displayed in a 2-D image is usually not an issue. In a 3-D projection, achieving good spatial perception can be difficult. Major influencing factors are, for example, shadows (Wanger et al., 1992) and illumination models (e.g. Weigle and Banks, 2008; Lindemann and Ropinski, 2011, and references therein). The issue is also noted by Szoke et al. (2003). As an approach, they have implemented a switch to an overhead view and a vertically moveable map in D3D to enable the forecaster to better judge the spatial position of a 3-D feature.

\subsection{Ensemble visualization}

Ensemble visualization aims at identifying variability, similarities and differences among ensemble members. It is closely related to uncertainty visualization, of which Pang et al. (1997) and Johnson and Sanderson (2003) provide early overviews. In the atmospheric sciences, 2-D visualizations of statistical quantities that summarize the ensemble distribution or that represent relative frequencies for events are frequently used. Wilks (2011, ch. 7.6.6) lists a number of techniques. For example, current products provided in ECMWF's "ecCharts" system (Lamy-Thépaut et al., 2013) include maps of mean and standard deviation (SD), maps of threshold probabilities (for example, the probability of precipitation exceeding a critical threshold) and of derived statistical measures (for example, the extreme forecast index; Lalaurette, 2003).

In a recent survey - also including applications outside the atmospheric domain - Obermaier and Joy (2014) classify ensemble visualization methods described in the literature into "location-based methods" and "feature-based methods". Location-based methods compare ensemble properties at fixed locations in the data set. In the simplest case, this includes the ensemble mean, SD or probability as computed at a given grid point. Such statistical quantities have been visualized via colour maps, opacity, texture and animation (Djurcilov et al., 2002; Rhodes et al., 2003; Lundstrom et al., 2007). Also, glyphs have been used to display, for example, uncertainty in wind fields (Wittenbrink et al., 1996). Featurebased methods, on the other hand, extract features from each ensemble member and aim at visually comparing the de- 
tected features. Examples include spaghetti plots (where the isolines are the features), the joint display of detected cyclonic features (Hewson and Titley, 2010) and visualization techniques for the prediction of hurricane tracks (Cox et al., 2013). Recently, Whitaker et al. (2013) have generalized box plots to contour box plots to enable an improved quantitative and qualitative analysis of ensembles of 2-D isocontours and level sets. In 3-D, the effect of uncertainty on the position of 3-D isosurfaces has been the topic of a number of studies. It has been approached with, for instance, geometric displacements (Grigoryan and Rheingans, 2004) and surface animation (Brown, 2004). In a study concerning the reconstruction of the Earth's subsurface model, Zehner et al. (2010) visualize confidence intervals around an isosurface using additional transparent surfaces as well as lines connecting the surfaces. Recently, techniques have used stochastic modelling of uncertainty in scalar ensembles to quantify and visualize the possible occurrences of isosurfaces (Pöthkow and Hege, 2011; Pöthkow et al., 2011; Pfaffelmoser et al., 2011; Pfaffelmoser and Westermann, 2012). The latter studies all include examples from the atmospheric domain.

A few articles in the visualization literature have presented software tools that put special emphasis on ensembles in earth science applications. Potter et al. (2009) present the "Ensemble-Vis" tool and investigated the usage of multiple linked views to visualize 2-D weather simulation ensembles. They conclude that the combination of standard statistical displays (spaghetti plots, maps of mean and SD) with user interaction facilitates clearer presentation and simpler exploration of the data. In their "Noodles" tool, Sanyal et al. (2010) enhanced spaghetti plots by glyphs and confidence ribbons to highlight the Euclidean spread of 2-D contour ensembles. They describe the usage of their methods by atmospheric researchers investigating different parametrizations in the Weather Research and Forecasting (WRF) model. Sanyal et al. (2010) also highlighted the positive effect of interactivity and linked views on the user and note the challenge of potential generalization of their work to three dimensions. Recently, Höllt et al. (2014) have presented "Ovis", a system for the visualization of 2-D ocean height-field ensemble data. They again use linked views of maps, statistical plots and 3-D renderings and demonstrate the use of time-series glyphs for the comparative visualization of the ensembles at two different positions over time. Höllt et al. (2014) discussed the application of their tool to off-shore oil operations and the planning of underwater glider paths.

\section{The 3-D ensemble visualization tool Met.3D}

Met.3D has been developed to support ensemble data exploration during forecasting, in particular for field campaigns (at the time of writing this paper). Beside this primary objective, we have designed the software in a way that it can be used as a framework into which new ensemble visualization

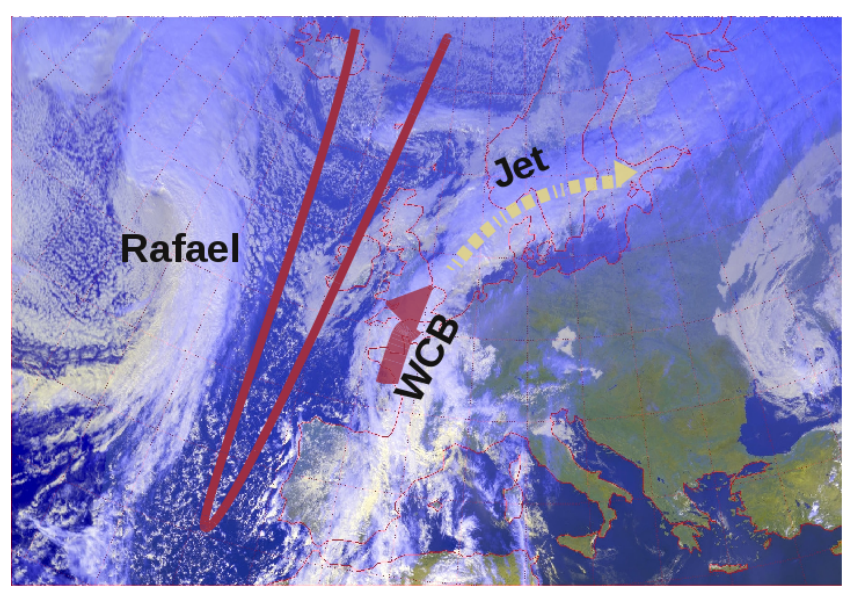

Figure 1. Real-world context for the T-NAWDEX-Falcon case used for the examples: visible Meteosat satellite image of Europe and the North Atlantic of 12:00 UTC, 19 October 2012 (Meteosat operated by EUMETSAT, image processing by DLR-IPA). Important features are the narrow trough to the west of the British Isles (dark red line), the former Hurricane Rafael and the WCB manifest in the cloud band east of the trough.

techniques can be implemented and evaluated with respect to their use in forecasting. We note that Met.3D is not intended to be a full-featured meteorological workstation; this would be beyond the scope of our work.

At the time of writing, Met.3D supports forecast data from the ECMWF Ensemble Prediction System (ENS), comprising 50 perturbed forecast runs and an unperturbed control run (Buizza et al., 2006; Miller et al., 2010). These 51 forecast members approximate the distribution of possible future weather scenarios (Leutbecher and Palmer, 2008).

The visualization examples shown in this paper use data from the TNF forecast case of 19 October 2012. The satellite image in Fig. 1 provides a real-world observation of major features that appear in the visualizations: a distinct narrow trough was located to the west of the British Isles. Upstream of the trough the former Hurricane Rafael transformed into a strong mid-latitude cyclone. East of the trough, ascending WCB air masses formed a cloud band extending from Spain to the British Isles. The clouds further stretch along a jet stream over southern Scandinavia and the Baltic Sea.

The static images shown in the following sections are complemented by video clips contained in the Supplement to this paper, helping to illustrate the interactive capabilities of Met.3D. The videos are screen recordings realized on hardware consisting of a consumer-class six-core Intel Xeon running at $2.67 \mathrm{GHz}$, equipped with $24 \mathrm{~GB}$ of RAM, a $512 \mathrm{~GB}$ solid state drive and an Nvidia GeForce GTX 560Ti graphics card with $2 \mathrm{~GB}$ of video memory. 


\subsection{User interface}

Figure 2 shows the graphical user interface (GUI) of Met.3D. The forecast data fields can be displayed in multiple 3-D views (Fig. 2a, b, c). In the horizontal, a cylindrical longitude-latitude projection is used. As common in meteorology, the logarithm of pressure serves as the vertical coordinate. Vertical scale, i.e. the proportion of vertical to horizontal units, can be specified for each view individually. Time navigation is provided for the forecast initialization (or base, or run) time and the forecast valid time (Fig. 2d). This way, subsequent forecast runs can be checked for consistency by keeping the valid time fixed and changing the initialization time. A distinct feature is the ensemble navigation. The user can select a specific forecast member for exploration, animate over members and toggle the ensemble mean for all currently displayed data fields (Fig. 2e).

Visual entities such as a horizontal or vertical cross section, the base map or a 3-D isosurface are represented by "actors" and are assigned to a "scene". A scene, in other words a collection of actors, can be assigned to one of the views for rendering. An actor can be part of multiple scenes. For example, a cross section could be viewed as a traditional 2-D image in one view, and be combined with a 3-D isosurface in another. If the section is relocated, its position is updated in both views. To keep the user interface simple, properties that the user can modify for a particular actor (e.g. the isovalue of an isosurface, the forecast variable displayed by an actor, the associated colour palette) are arranged in a tree-like structure on the left of the Met.3D window and are easily accessible (Fig. 2f). If used in a forecast setting, only the uppermost tree nodes are required by the user to, for instance, load predefined forecast products.

Trafton and Hoffman (2007) point out the importance of visual comparisons in the forecasting process. Met.3D's actors can be synchronized in time and ensemble dimension, its views can be synchronized to the same camera viewpoint. Thus, side-by-side comparison of different data sets is facilitated.

\subsection{A bridge from 2-D to 3-D}

To help forecasters transition to the 3-D visualization environment, we have implemented horizontal and vertical 2-D sections. The sections reproduce the look of the corresponding products in the DLR MSS (Rautenhaus et al., 2012), providing filled and line contours, wind barbs, coast lines and graticule. In Met.3D, the sections are embedded into the 3-D context and can be interactively moved in space by the user in real time. This provides a very fast means to explore the atmosphere's vertical structure (by sliding a horizontal section up and down), or the change in forecast variables along a flight track when a waypoint is relocated (by moving a vertical section). Also, the camera can be moved interactively to zoom in, pan or tilt the view - for instance, to view multi- ple sections stacked on each other from an angled viewpoint. Figure 3 illustrates the concept. The forecast wind field is visualized by means of a horizontal and vertical section. The horizontal map - largely resembling the corresponding product from the MSS - is stacked on top of surface level contours displaying the mean sea level pressure (Fig. 3b). The vertical section is augmented by a 3-D isosurface of wind speed (Fig. 3c); the isovalue is chosen such that the strongest winds of the jet stream, an important indicator for the large scale flow of the upper troposphere, are captured. The 3-D display allows us to locate the vertical section in space and additionally provides information on the spatial structure of the jet.

We approach the challenge of spatial perception by drawing projections of all rendered structures to the surface to imitate shadows generated by a light source above the scene. As illustrated in Fig. $3 \mathrm{~b}$ and c, the shadows help to qualitatively judge the elevation of a feature, and also show its horizontal location. To improve the quantitative judgement of elevation, the user can colour the isosurface according to pressure elevation, and place vertical poles in the scene that provide labelled pressure axes (Fig. 3c). The poles can be interactively moved in the scene (by picking and dragging handles that appear in an "interaction mode"), so that different locations can be probed.

Vertical sections can be drawn along an arbitrary number of waypoints (Fig. 3c). Analogous to vertical poles, each waypoint and section segment displays a handle in interaction mode that the user can drag to move the waypoint or segment. They can also be moved synchronously in multiple scenes, as illustrated in Fig. 4. Displayed are sections of potential vorticity (Fig. 4a, the red colours around values of 2 PVU (potential vorticity unit) show the dynamic tropopause) and cloud cover fraction (Fig. 4b). Wind barbs overlain on a horizontal section can be configured to automatically scale in size and density. In Fig. 5, the horizontal section of equivalent potential temperature shows the different character of air masses transported by Rafael. When the user zooms into the view, Met.3D increases the density of the wind barbs (Fig. 5b). The frontal zone along which the typical change in wind direction occurs can now be well perceived.

With respect to colours used in the visualizations, it is important to address perceptual issues (Hoffman et al., 1993). To map scalar value to colour, we have implemented the perceptually based hue-chroma-luminance (HCL) colour space. Following Zeileis et al. (2009) and Stauffer et al. (2015), the user can create colour palettes by specifying ranges in hue, chroma and luminance. Alternatively, colours can be explicitly specified to reproduce colour bars the user is familiar with. An example is the colour palette for potential vorticity shown in Fig. 4. 


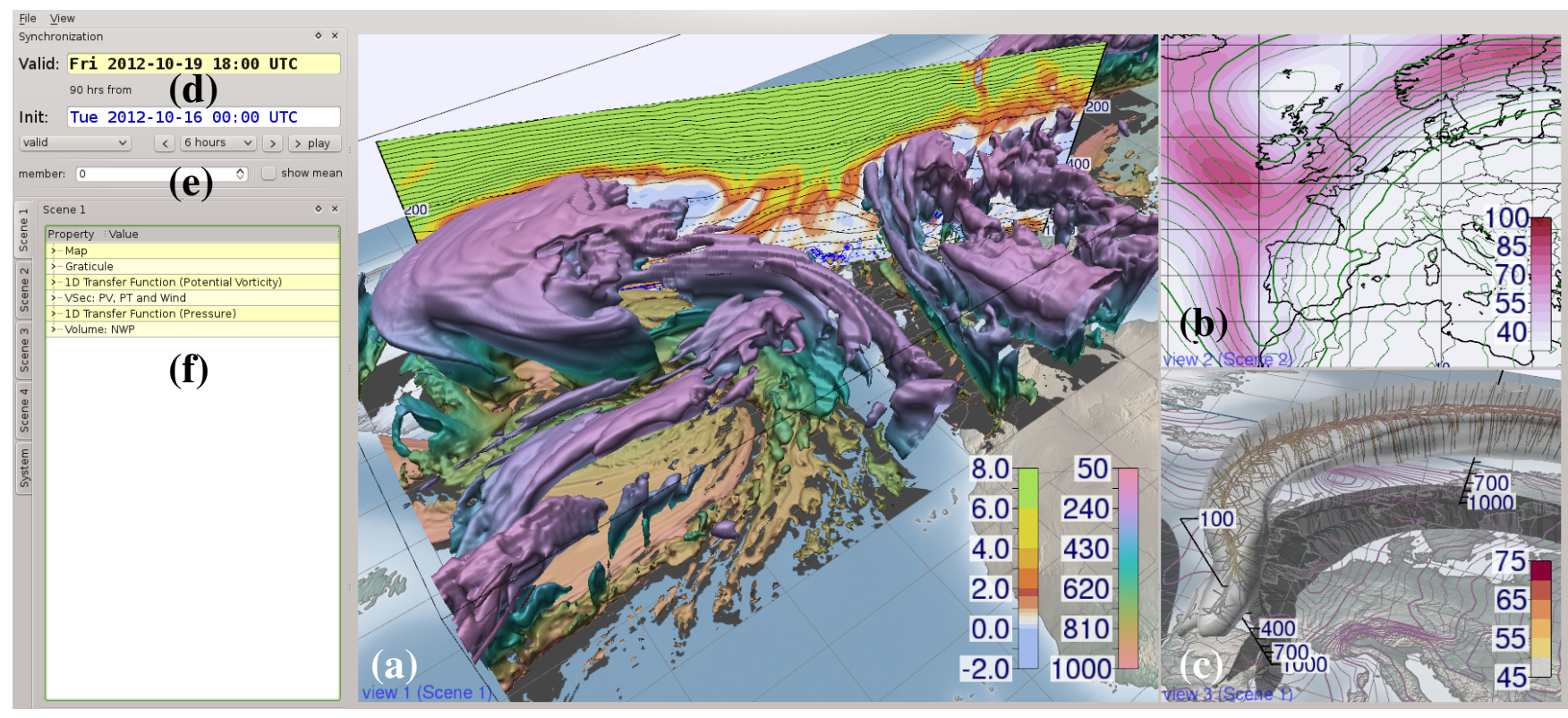

Figure 2. The main user interface of Met.3D. We apply 2-D and 3-D visualization techniques to explore ensemble weather forecasts. (a) Isosurfaces of cloud cover fraction of 0.5 coloured by elevation $(\mathrm{hPa})$, and a vertical section of potential vorticity (PVU). (b) Horizontal section with contour lines of the mean geopotential height field $(\mathrm{m})$ and filled contours of its SD (m). (c) Normal curves applied to the wind field to visualize the jet core. The white isosurface shows $45 \mathrm{~m} \mathrm{~s}^{-1}$. Colour coding in $\mathrm{m} \mathrm{s}^{-1}$. (d-f) See text for details.

\subsection{Ensemble support}

Met.3D enables the forecaster to explore variation in the ensemble, to identify regions in which the forecast is uncertain, and to explore possible forecast scenarios. The user can interactively navigate through the ensemble members to judge the variability in the forecast. Each member can also be explored individually. Statistical measures including threshold probabilities, mean, minimum, maximum and SD can be derived on demand. For threshold probabilities (for example, wind speed exceeding $45 \mathrm{~m} \mathrm{~s}^{-1}$ or cloud cover fraction being below 0.2 ) the threshold value can be adjusted interactively.

Figure 6 shows an example of exploring the upper-level ensemble wind field of the forecast from Monday, 15 October 2012, 00:00 UTC, valid at Friday, 19 October 2012, 18:00 UTC. To visualize the jet stream, two wind speed isosurfaces are rendered. The large variation of the ensemble regarding position, structure and strength of the jet stream over the Atlantic highlights high uncertainty in this area. On the other hand, the strong jet extending from Spain to Scandinavia is predicted with higher certainty; while in the mean wind field the $45 \mathrm{~m} \mathrm{~s}^{-1}$ signal over the Atlantic is largely smoothed out, it is present over Europe (Fig. 6d). However, adding a horizontal section of wind speed SD (Fig. 6e) to the isosurface of mean wind speed reveals that the position of the jet is uncertain in particular on its northern side.

Figure 7 shows the probability of wind speed exceeding $45 \mathrm{~m} \mathrm{~s}^{-1}$. A high probability of over $70 \%$ can again be found over northern Europe (Fig. 7a). The large horizontal extent of the area of low (10\%) probability above the Atlantic reflects the uncertainty. The actual jet can occur anywhere in this region. Two days later, with decreasing forecast lead time, the ensemble has significantly converged and the uncertainty has decreased (Fig. 7b).

Figure 7c and d show the probability of the SchmidtAppleman criterion (Schumann, 1996), an indicator for the occurrence of contrails (aircraft-induced clouds that also have been the target of research flights; Voigt et al., 2010; Kaufmann et al., 2014). Visualization of the probability of the Schmidt-Appleman criterion being fulfilled shows that contrails, in the example, can only occur between about 400 and $200 \mathrm{hPa}$. In the given case, a high probability can be observed on the leading downstream edge of the jet.

\subsection{Normal curves}

In the volume visualizations shown in Figs. 6 and 7, the structure of the scalar fields inside the transparent isosurfaces cannot easily be inferred. As stated in Sect. 2.1, this is a disadvantage of 3-D visualization: while an isosurface allows for inference on the 3-D spatial structure of the displayed data field, it only displays a single data value. Although two or three isosurfaces can be rendered in a single image using transparency, the image quickly becomes illegible when more surfaces are used. "Normal curves" were suggested by Pfaffelmoser et al. (2011) to estimate the spatial distance between two isosurfaces. For our application, we propose to use "3-D normal curves" as an intermediate means between 

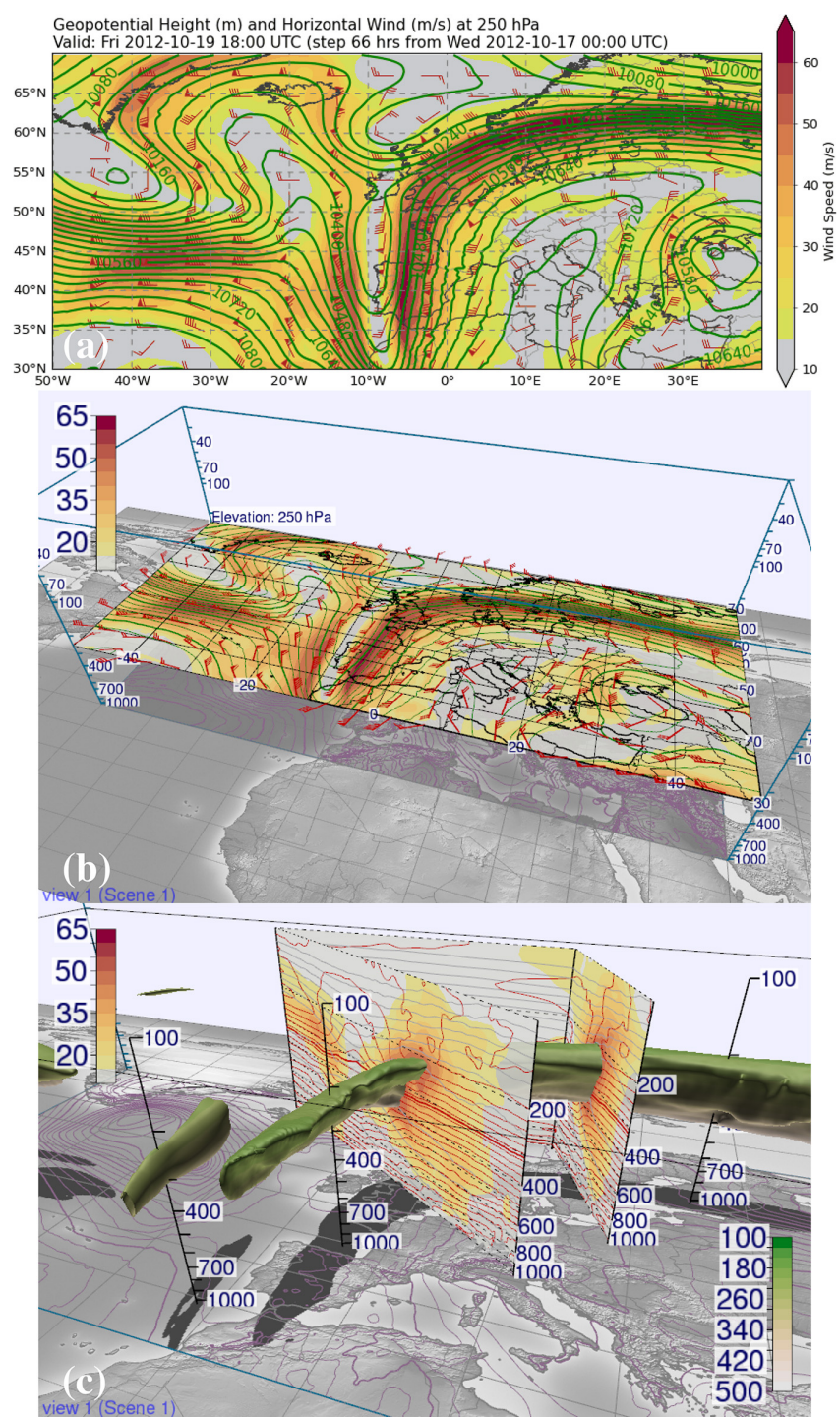

Figure 3. Bridge from 2-D to 3-D visualization. (a) Horizontal section of geopotential height (contour lines) and horizontal wind speed (colour) at $250 \mathrm{hPa}$, as obtained from the DLR Mission Support System. ECMWF deterministic forecast from 00:00 UTC, 17 October 2012, valid at 18:00 UTC, 19 October 2012. (b) The same data, rendered by Met.3D and mapped into the 3-D context. The section can be interactively moved by the user. (c) Vertical section of horizontal wind speed (colour) and potential temperature (contour lines) in Met.3D, amended by a $50 \mathrm{~m} \mathrm{~s}^{-1}$ isosurface of wind speed, coloured by pressure $(\mathrm{hPa})$. Note how spatial perception of the 3-D isosurface is aided by rendering shadows and labelled vertical poles (animated version of this figure in the Supplement at 00:05 $\mathrm{min}$ ).

a 2-D section and a 3-D isosurface to visualize the structure of scalar fields in the interior of an isosurface.

The curves are started on a transparent isosurface and proceed along the field's gradient direction, i.e. normal to the isosurface. The spacing of the curves can be controlled by the user (cf. Sect. 4.4). We colour the curves according to the scalar value. This way, we achieve a visual sampling of a subdomain of the volume. In contrast to a 2-D section that samples a planar subdomain, the normal curves sample a 3-D subdomain enclosed by an isosurface via a discrete set of lines. Following the gradient, the curves converge at local extrema of the data field. This way, the user can at a glance identify the locations and strengths of present extrema, and judge the strength and direction of the gradient between an extremum and the outer isosurface.

Figure 8 illustrates the approach. The goal is to identify regions of maximum probability of cloud ice water content exceeding $0.01 \mathrm{~g} \mathrm{~kg}^{-1}$, and to track the regions' evolution over time. The normal curves immediately show a maximum in the upper part of the transparent $40 \%$ isosurface (Fig. $8 \mathrm{~b}$ and c). The corresponding shadows reveal that the maximum is approximately located above the Pyrenees. Interaction with the vertical axis shows a vertical position between 300 and $200 \mathrm{hPa}$. Further visual aids can now be added to obtain more quantitative information. In the example, the horizontal section can be immediately placed in the region of interest, without the need to search the entire vertical extent of the model atmosphere (Fig. 8d).

While extrema can also be identified with an inner opaque isosurface (cf. Fig. 7) or by interacting with 2-D sections, the normal curve approach requires less interaction steps. This is advantageous if the absolute values of the extrema are not known beforehand (with isosurfaces the user needs to search over isovalues), and if the extrema shall be visually tracked over ensemble members or time. Concerning time, in particular probability values tend to decrease with increasing forecast lead time; hence, a fixed isosurface is not well suited to visualize the temporal evolution of a maximum.

In Fig. 2c (also shown in the video at 05:40 min), the method is applied to the upper-level wind field shown in Fig. 6. Here, the normal curves inside the $45 \mathrm{~m} \mathrm{~s}^{-1}$ isosurface converge to the string-like line of local maxima in the wind field - the curves are used to identify the position of the jet core and its strength.

\section{Visualization algorithms and system architecture}

Response time, the time required to display a new image after the user has interacted with, for example, camera or time step, is crucial to the acceptance of an interactive visualization tool, as Szoke et al. (2003) and Hibbard (2004) emphasize. To achieve low response times, we make extensive use of modern graphics processing units (GPUs). These highly parallel processors provide high computational throughput and memory bandwidth and are well suited to accelerate visualization algorithms.

GPU acceleration is implemented with OpenGL 4 and the OpenGL Shading Language (GLSL) ${ }^{1}$, using vertex, geom-

\footnotetext{
${ }^{1}$ https://www.opengl.org/documentation/glsl/
} 

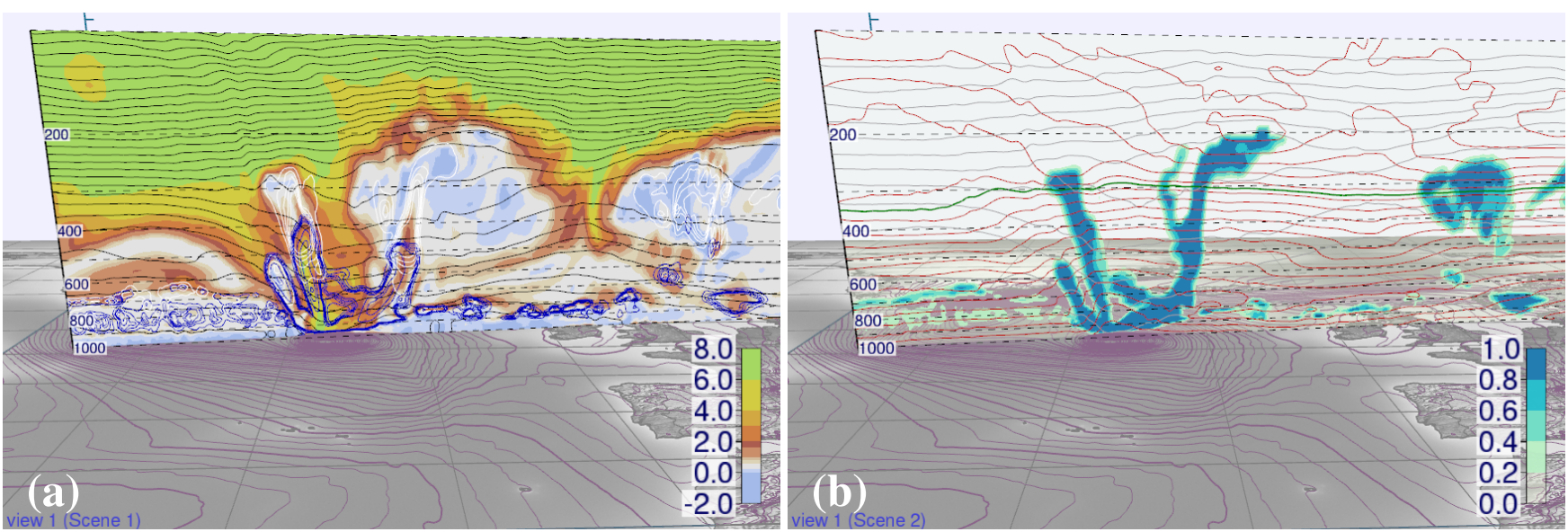

Figure 4. Vertical sections can be moved interactively in Met.3D to explore the vertical structure of the atmosphere, for example along potential flight track segments. (a) Potential vorticity (colour coding in PVU), (b) cloud cover fraction. Red colours in (a) mark the 2-PVU surface and thus the dynamic tropopause. Note the low tropopause along the trough. Same forecast as in Fig. 3 (animated version of this figure in the Supplement at 01:24 min).
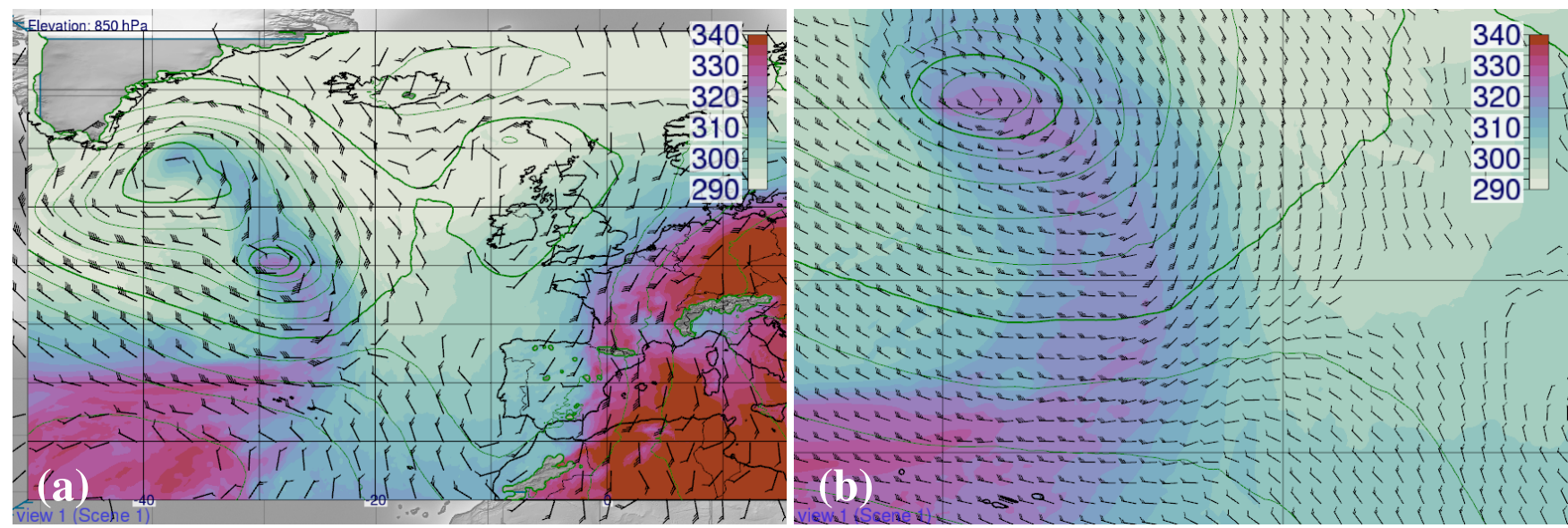

Figure 5. Met.3D automatically scales size and density of wind barbs overlain on horizontal sections. (a and b) Equivalent potential temperature (colour coded in $\mathrm{K}$ ) at $850 \mathrm{hPa}$, overlain with contour lines of geopotential height. Same forecast as in Fig. 3 (animated version of this figure in the Supplement at 01:54 min).

etry, fragment and compute shaders. These small GPU programs allow the parallel execution of operations on the level of a graphics vertex or of an output fragment (i.e. a single pixel in the generated image), the generation of new geometry by the graphics subsystem, or the general parallel execution of operations. We will not go into detail of graphics technology here, for an introduction to GPU-based visualization we refer the reader to, for example, Bailey $(2009,2011$, 2013) or Engel et al. (2006). On the CPU side, Met.3D is implemented in $\mathrm{C}++$.

A second important factor influencing response time is the way data are read from disk and whether and how it needs to be processed prior to visualization. We have designed an ensemble data pipeline to handle this task efficiently.
In this section, we discuss the methods used to achieve high visualization performance in Met.3D. After describing the data that can be handled by the tool (Sect. 4.1), we discuss the ensemble data pipeline (Sect. 4.2) and the GPU-based visualization algorithms (Sect. 4.3 and 4.4).

\subsection{Forecast data}

The data upon which we have based our visualization methods are obtained from the ECMWF global ensemble weather prediction system ENS and the high-resolution deterministic integrated forecast system (IFS). One of our system design goals was to support the forecast data in the format they can be retrieved from the ECMWF Meteorological Archive and Retrieval System (MARS). MARS outputs the data interpo- 

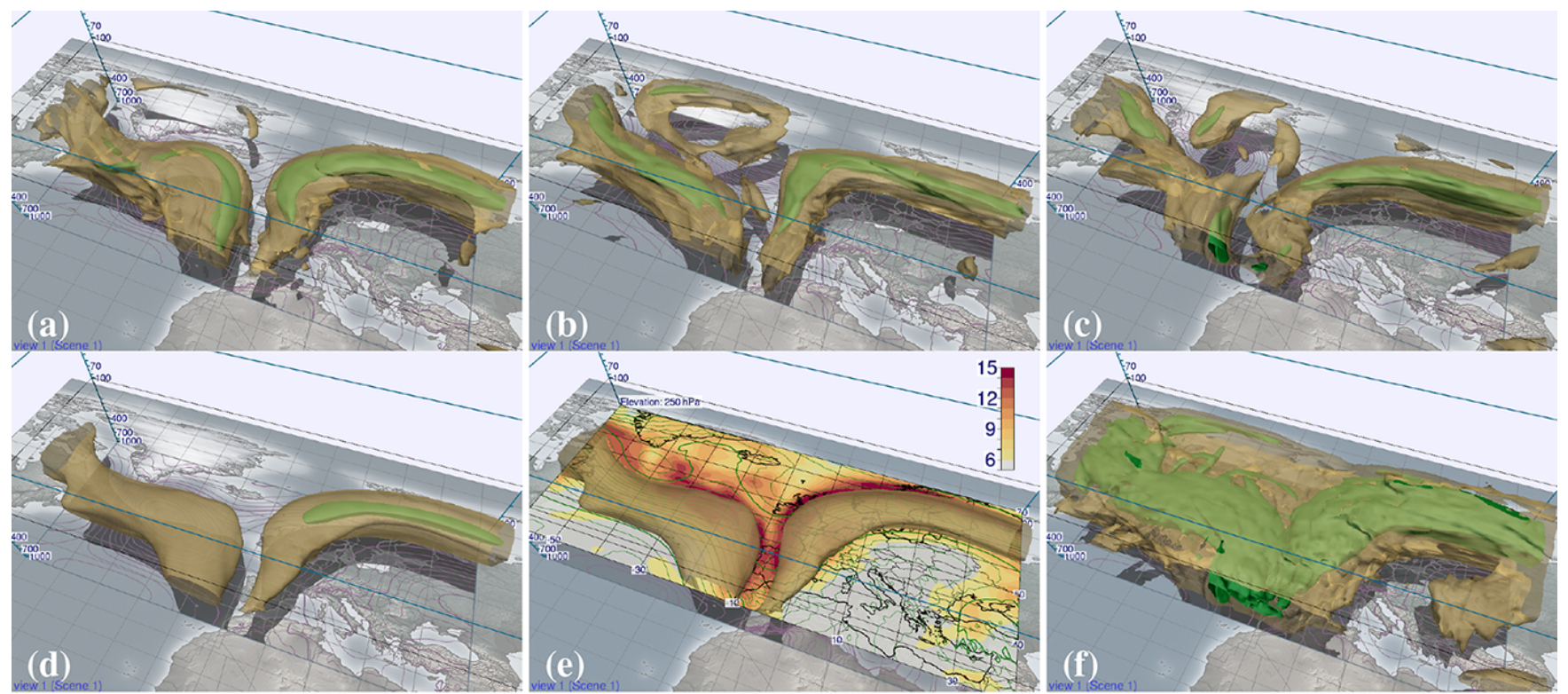

Figure 6. Navigation through the ensemble. Visualized are the $50 \mathrm{~m} \mathrm{~s}^{-1}$ (green opaque) and $30 \mathrm{~m} \mathrm{~s}^{-1}$ (yellow transparent) isosurfaces of horizontal wind speed (forecast from 00:00 UTC, 15 October valid at 18:00 UTC, 19 October 2012). (a) Control run, members (b) 27 and (c) 33, (d) ensemble mean, (e) ensemble mean augmented by a horizontal section of SD ( $\mathrm{m} \mathrm{s}^{-1}$ ), (f) ensemble maximum (animated version of this figure in the Supplement at 02:26 min).

lated in the horizontal to a regular latitude-longitude grid. In the vertical, the data are available on either a set of predefined pressure levels (PLs), or, higher resolved and thus better suited for 3-D visualization, on the native model grid levels (MLs). For the latter, the model uses terrain following hybrid sigma-pressure coordinates, as illustrated in Fig. 9. The vertical-pressure coordinate $p_{k}$ of a grid point at level $k$ is defined by a set of fixed coefficients $a_{k}$ and $b_{k}$ and the surface pressure $p_{\text {sfc }}$ below the grid point (Untch and Hortal, 2004): $p_{k}=a_{k}+b_{k} \times p_{\text {sfc }}$. With increasing altitude the influence of $p_{\text {sfc }}$ decreases. During TNF, the operational ensemble forecast was available with 62 levels (91 levels for the deterministic forecast, increased by the time of writing to 137 levels). At this resolution, levels are constant in pressure above approximately $64 \mathrm{hPa}(70 \mathrm{hPa})^{2}$. In the horizontal, a spectral truncation of T639 (T1279) is available, corresponding to a regular latitude-longitude grid of approx. $0.28^{\circ} \times 0.28^{\circ}\left(0.15^{\circ} \times 0.15^{\circ}\right)$. Forecasts are available twice daily (starting at 00:00 and 12:00 UTC) at a time step of $3 \mathrm{~h}$ up to $144 \mathrm{~h}$ forecast lead time and $6 \mathrm{~h}$ up to $240 \mathrm{~h}$ forecast lead time.

For the examples in this paper, we use ENS data interpolated horizontally to $1^{\circ} \times 1^{\circ}$ and to $0.25^{\circ} \times 0.25^{\circ} ; 1^{\circ} \times 1^{\circ}$ is the grid spacing we were able to operationally retrieve during $\mathrm{TNF}$, as permitted by the available internet bandwidth and interpolation time required by MARS. Deterministic data are used at $0.15^{\circ} \times 0.15^{\circ}$ grid spacing. In the vertical, all 62 and 91 levels are used.

\footnotetext{
${ }^{2}$ http://old.ecmwf.int/products/data/technical/model_levels/
}

The forecast domain used in the examples encompasses $100^{\circ}$ in longitude by $40^{\circ}$ in latitude, resulting in $101 \times 41 \times 62$ grid points for ENS data fields at $1^{\circ} \times 1^{\circ}$ grid spacing, $401 \times 161 \times 62$ points at $0.25^{\circ} \times 0.25^{\circ}$ grid spacing and $669 \times 268 \times 91$ points for the deterministic forecast at $0.15^{\circ} \times 0.15^{\circ}$ grid spacing. Using floating point precision (4 bytes per value), the data fields require approximately 1 , 16 and $62 \mathrm{MB}$ per member, time step and forecast parameter in graphics memory. For visualizations using multiple forecast parameters and the entire ensemble, the required memory quickly adds up.

Forecast data can be read directly from GRIB files output by MARS or from NetCDF-CF ${ }^{3}$ files. Our goal was to minimize the time span between data availability at ECMWF and visualization. Hence, no pre-processing of the data prior to usage in Met.3D is required. Forecast parameters not output by the ECMWF model, however, need to be computed first. For this purpose, Met.3D can be connected to the data processing system of the DLR MSS, which derives additional quantities (for example, relative humidity and potential vorticity) from the forecast parameters output by ECMWF.

\subsection{Ensemble processing pipeline}

To process the ensemble data prior to rendering, we have designed a data processing pipeline composed of modules ("data sources") that create, read or process data and that can be combined in flexible ways. Figure 10 illustrates the con-

\footnotetext{
${ }^{3}$ http://cfconventions.org/
} 


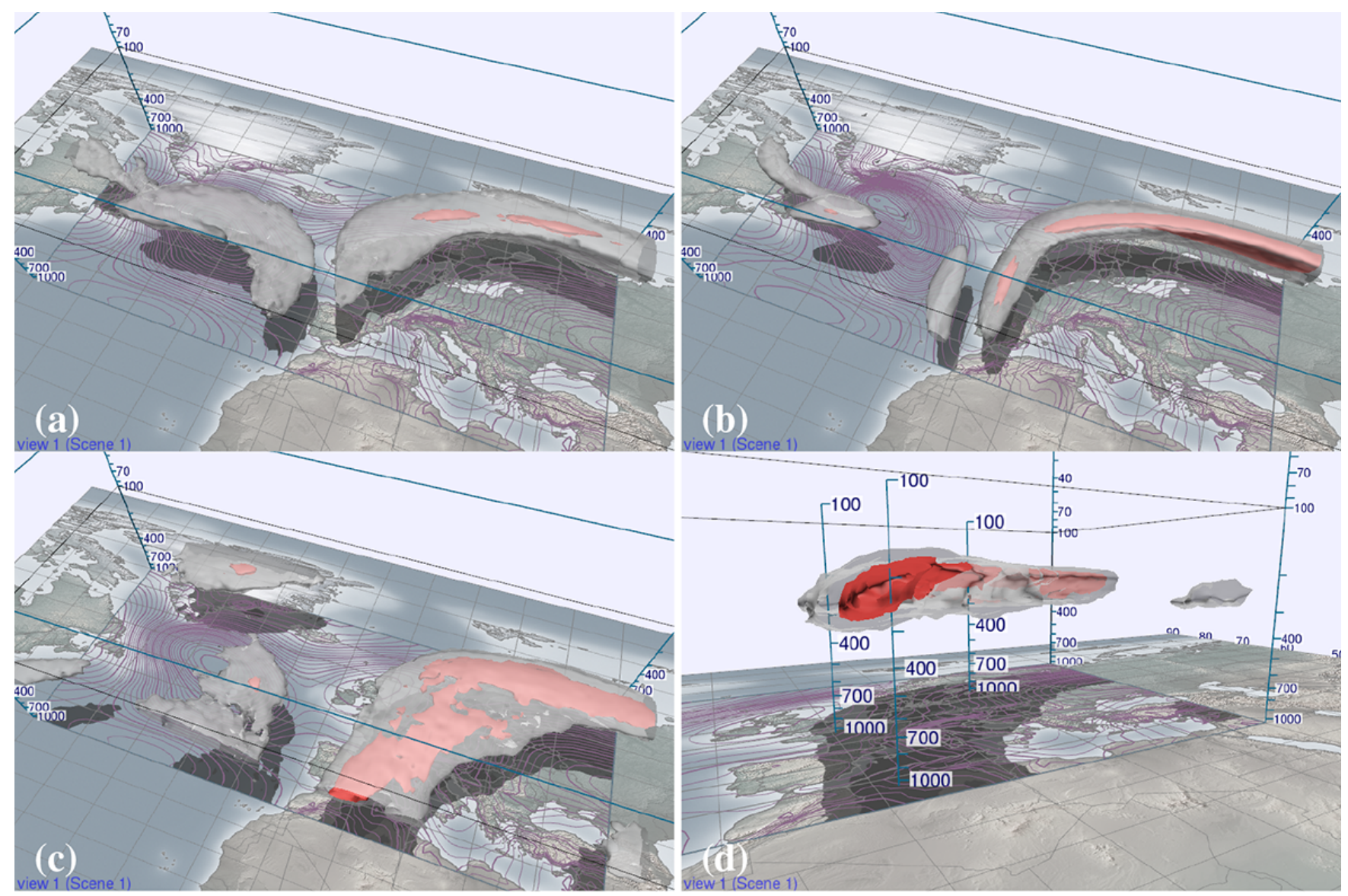

Figure 7. Probability fields computed from the ensemble, valid at 18:00 UTC, 19 October 2012. (a and b) Probability of horizontal wind speed exceeding $50 \mathrm{~m} \mathrm{~s}^{-1}$, as computed from the forecast initialized (a) at 00:00 UTC, 15 October 2012 and (b) at 00:00 UTC, 17 October 2012. Shown are the $70 \%$ (red opaque) and $10 \%$ (white transparent) isosurfaces. Note how the ensemble converges. (c and d) Probability of contrail occurrence (Schmidt-Appleman criterion fulfilled and relative humidity greater than $80 \%$ ), as viewed from different camera positions (80\% red opaque and 50\% white transparent) (animated version of this figure in the Supplement at 03:23 min).

cept. Algorithms in the data sources (for example, ensemble statistics or trajectory filtering; cf. Part 2) can be implemented to execute on either CPU or GPU (the latter via compute shaders). All data sources are connected to a memory manager that caches intermediate results. The actors that implement the visualization methods are placed at the end of a pipeline. They send "requests" into the pipeline to obtain a specific data item. These requests are composed of multiple key/value pairs similar to the Web Map Service requests used in the MSS (see Rautenhaus et al., 2012, for details). A request emitted into a pipeline propagates from data source to data source. Each data source interprets the keys it requires. If the requested operation has been executed before and the result has been cached, no action is taken. Otherwise, the data source defines a processing task to perform the requested operation. The task, however, is not executed immediately. If applicable, remaining keys are passed on to the data source's input(s). If a data source requires additional input, it can also append keys to the request.
All processing tasks defined this way are assembled into a task graph that is passed to a scheduler for execution. Based on the dependencies provided by the graph structure and information carried by the tasks, the scheduler can process the tasks. For example, tasks that have to be performed for all members of the ensemble can be executed in parallel.

As an example, consider the pipeline depicted in Fig. 10b. The volume actor at the end of the pipeline emits a request for a scalar field containing the probability of horizontal wind speed exceeding $45 \mathrm{~m} \mathrm{~s}^{-1}$. The module computing the probability field requires the wind field of each ensemble member, regridded to a common grid. Hence, requests for regridded data fields containing the members' wind speed are emitted and a task is set up to compute the probability from these fields. The regridding module, in turn, requests that the wind speed fields are read from disk by the reader module. For an ensemble of size $M$, the resulting task graph (Fig. 10c) contains $M$ tasks to read the wind field of a single member, $M$ tasks to regrid these fields to a common grid and one task 

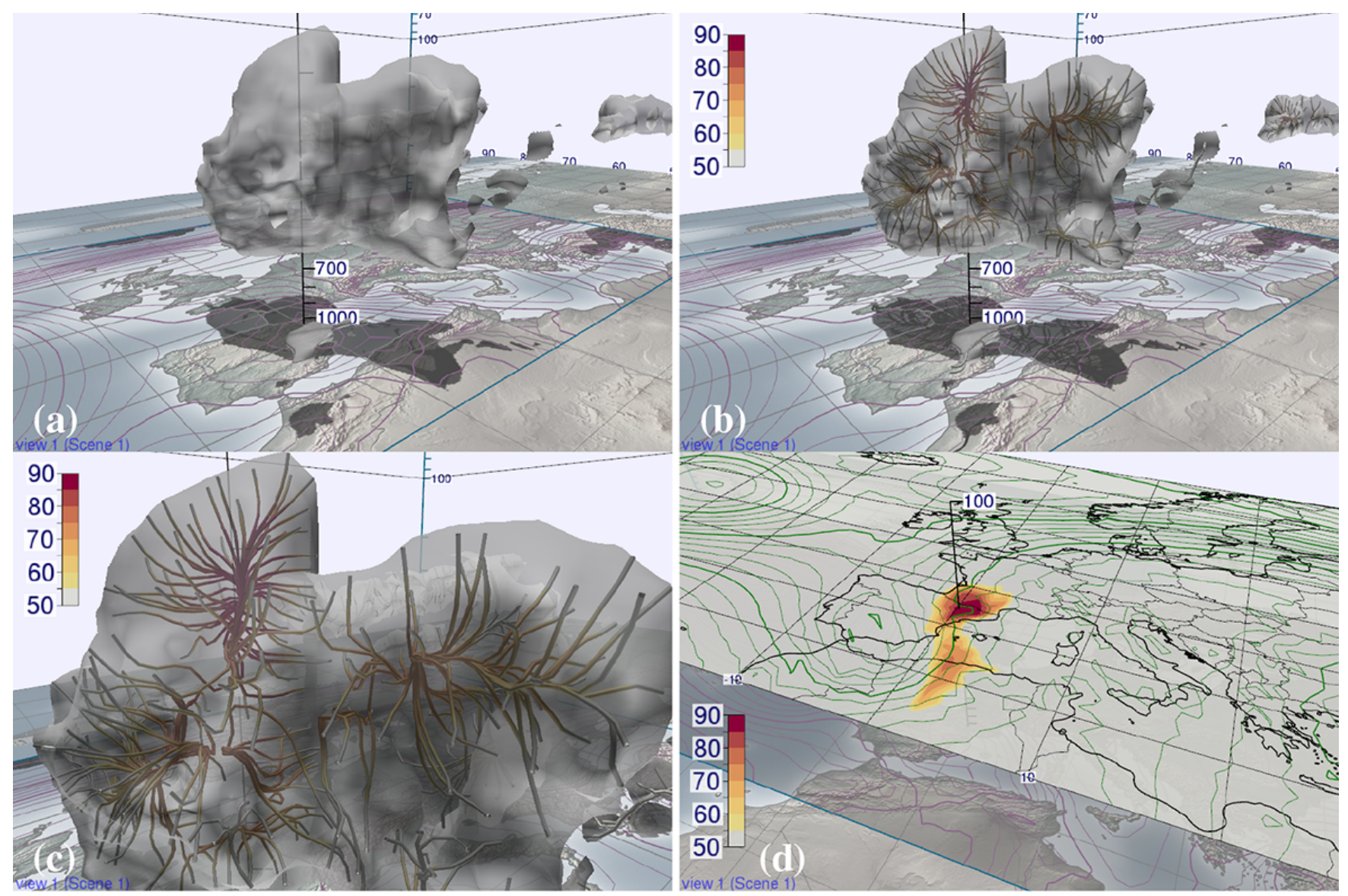

Figure 8. Normal curves help to analyse the topology of 3-D scalar fields. They reveal the distribution of data values in a subdomain enclosed by a 3-D isosurface and enable fast identification and tracking of local extrema. (a-c) Probability of cloud ice water content exceeding $0.01 \mathrm{~g} \mathrm{~kg}^{-1}$. The white transparent isosurface shows $40 \%$ probability. Colour coding in \%. (d) Details of the identified maximum are inspected with a horizontal section at $250 \mathrm{hPa}$. Forecast from 00:00 UTC, 17 October 2012 valid at 12:00 UTC, 20 October 2012 (animated version of this figure in the Supplement at 04:28 min).

to compute the probabilities. The regridding tasks are well suited to be executed in parallel.

To indicate an order of magnitude of the response times that Met.3D achieves on our test hardware when the displayed data field is changed, Table 1 lists timings for changing the forecast time in the horizontal section in Fig. 3. Timings are provided for displaying a single member of the ensemble and for displaying the ensemble mean (the latter as an example of a statistic that requires all members of all variables when computed on demand), both when data need to be read from disk and when it is available in cache. If the data to be visualized are available in cache, no task graph needs to be executed and the response time is of the order of a few milliseconds. If data need to be read from disk, the response time is bounded by the disk's bandwidth. This becomes noticeable in particular when ensemble statistical quantities are derived on demand. For the TNF data set at $0.25^{\circ}$ grid spacing, all members of the ensemble encompass approximately 3.2 GB that need to be read from disk. Our test hardware re- quires about $17 \mathrm{~s}$ for this task. One possibility to decrease this time is to pre-compute frequently used statistical quantities. In our set-up, this can be done with the MSS data processing system. However, the interactivity to change, for example, the threshold for a probability field is lost with this solution. Alternatively, the system performance can be increased by using pre-loading techniques to hide disk access. Here, the data for an anticipated subsequent time step are read in the background while the user explores the current time step. The current Met.3D architecture is prepared to implement such techniques. However, comprehensive optimizations of the system performance were outside the scope of this project and are left for future work.

\subsection{GPU-based visualization algorithms}

Met.3D's visualization algorithms support data fields on both hybrid sigma-pressure levels and on pressure levels. The difference is how the data fields are sampled on the GPU to obtain a value at a particular position in longitude-latitude- 


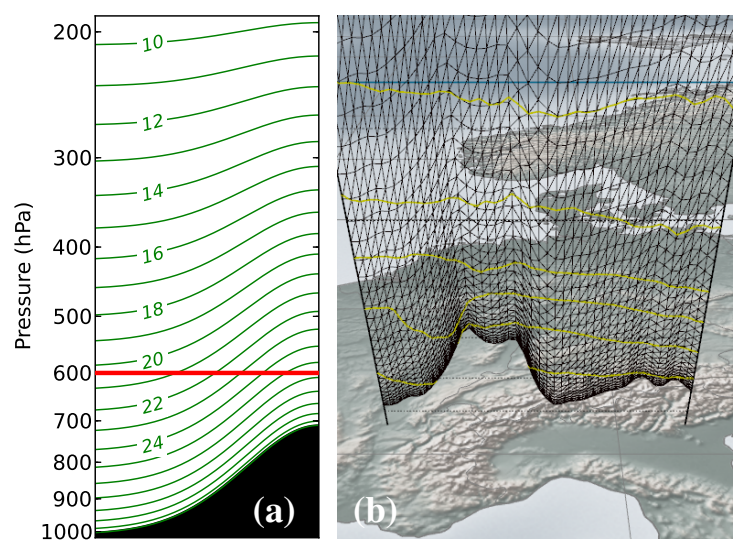

Figure 9. Hybrid sigma-pressure levels used by the ECMWF model. (a) The elevation of the model levels (green lines; the example shows levels from the 31 level model; level indices $k$ in green) changes with surface pressure (black curve at the bottom). The data value for a given pressure value $p$ can be located at different levels in the grid (the red line marks the location of $p=600 \mathrm{hPa}$ ). (b) Example of how the surface orography affects the vertical displacement of the grid points in a vertical section.

Table 1. Order of magnitude of response times achieved by Met.3D to display a new image after the user has advanced the forecast time for the horizontal section in Fig. 3b, displaying either data of a single member or of the ensemble mean (the latter an example of a statistic that requires all members of all variables when computed on demand). Timings are measured on the test hardware described in Sect. 3 and given for both forecast data at $1^{\circ}$ grid spacing and at $0.25^{\circ}$ grid spacing; 12 parallel threads are used by the scheduler for task graph execution. Fig. 3b uses four forecast variables, reading all ensemble members (for computation of the mean) from the disk hence involves reading $4 \times 51 \times 1 \mathrm{MB}$ at $1^{\circ}$ grid spacing and $4 \times 51 \times 16 \mathrm{MB}$ at $0.25^{\circ}$ grid spacing.

\begin{tabular}{llcc}
\hline Data & Source & $1^{\circ}$ & $0.25^{\circ}$ \\
\hline Single member & disk (NetCDF) & $50 \mathrm{~ms}$ & $365 \mathrm{~ms}$ \\
\hline Ensemble mean & disk (NetCDF) & $0.85 \mathrm{~s}$ & $17 \mathrm{~s}$ \\
\hline Single member & member in cache & $<10 \mathrm{~ms}$ & $25 \mathrm{~ms}$ \\
\hline Ensemble mean & mean in cache & $<10 \mathrm{~ms}$ & $25 \mathrm{~ms}$ \\
\hline
\end{tabular}

pressure space - an operation required by all visualization algorithms. In the horizontal, data fields on a regular longitude-latitude grid are supported.

To use the data on the GPU, a single forecast variable of a single member is stored in a 3-D texture (i.e. a 3-D data array) in GPU memory. We assume that these data fields fit into GPU memory. Longitude-latitude axes, as well as pressure levels for PL grids, are stored in an additional 1-D texture. For ML grids, the corresponding 2-D $p_{\text {sfc }}$ field and the coefficients $a_{k}$ and $b_{k}$ are stored. This allows for computation of the pressure coordinate of a grid point on the fly, without the need to use additional graphics memory for a 3-D texture with pressure values.

Horizontal 2-D sections on a pressure surface $p$ are rendered by placing the vertices of a grid of triangles horizontally at the positions of the data grid points and vertically at $p$ (Fig. 11a). Data sampling only needs to be done when $p$ is changed. Executed in parallel for each vertex, a binary search in the vertex shader yields the model levels (or pressure levels) $k$ and $k+1$ enclosing $p$ in the corresponding grid column. Following the ECMWF FULLPOS interpolation routines (Yessad, 2014), interpolation between these two levels is done linearly in $\ln (p)$. The results are cached in a 2-D texture. Filled contours are rendered by assigning colour to each fragment within a triangle in the fragment shader, using the horizontally hardware-interpolated scalar value. To obtain a colour, colour palettes (cf. Sect. 3.2) are stored as 1-D transfer functions in 1-D textures. These textures are used as lookup tables (LUTs), mapping a scalar value to a colour. Line contours are generated by a marching squares (e.g. Hansen and Johnson, 2005, ch. 1) implementation in a geometry shader. Each grid cell of the cached 2-D cross section texture is examined in parallel and, if applicable, a line segment is drawn. Graticule, coast and border lines are overlain on each horizontal section to improve spatial perception (cf. Fig. 3b). Wind barbs are also generated in a geometry shader. It takes the horizontal wind field's $u$ and $v$ components as input and generates the geometry of the barbs, again exploiting GPU parallelism.

Vertical sections are rendered with a similar grid of triangles. A triangle vertex is drawn for each vertical (model or pressure) level and each of a number of intermediate horizontal points along a line connecting the waypoints the user has specified (Fig. 9b). The distance between the intermediate points can be specified. A vertex shader computes the vertical position of each vertex and places it accordingly. This operation is a simple lookup for PL data and involves interpolation of $p_{\mathrm{sfc}}$ and computation of the model level pressure for ML grids. Scalar values are interpolated horizontally, also in the vertex shader, on the level on which the vertex is placed. They are also cached in a 2-D texture that is updated if a waypoint is moved. Filled and line contours are generated equivalently to those in the horizontal sections.

Three-dimensional isosurfaces are rendered with front-toback raycasting (Krüger and Westermann, 2003; Engel et al., 2006) implemented in the fragment shader. For each fragment (pixel) of the output image, a ray is cast through the data volume, sampling it at regular intervals and thus finding isosurface crossings. For this type of visualization algorithm, sampling the scalar volume is more expensive, as we need to interpolate in all three spatial dimensions to an arbitrary position in longitude-latitude-pressure space. For PL data, the grid is rectilinear (Fig. 11b) and can be sampled using texture mapping (e.g. Bailey, 2009), thus benefiting from the fast trilinear hardware interpolation provided by modern GPUs. By mapping the longitude-latitude-pressure coordinates of the 

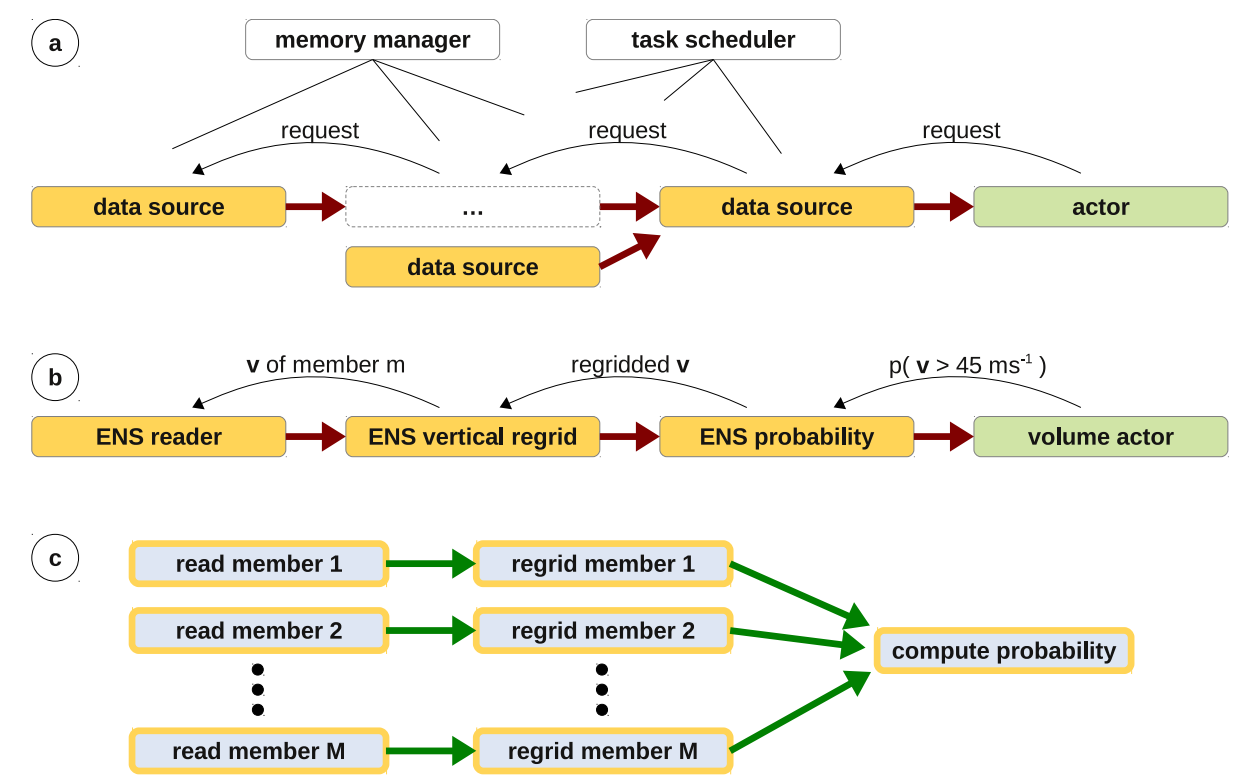

Figure 10. Pipeline concept of Met.3D: (a) data sources are connected to form a pipeline, into which a visualization "actor" sends data requests; (b) sample pipeline to visualize the probability of horizontal wind speed exceeding $45 \mathrm{~m} \mathrm{~s}^{-1}$. A request for the probability triggers further requests up the pipeline; (c) Task graph generated by the pipeline in (b).

sampling position to texture coordinates $\left(t_{\text {lon }}, t_{\text {lat }}, t_{p}\right)$ on the unit cube, the GPU interpolates the 3-D texture at an arbitrary position. For regular grids, this mapping is a simple linear scaling. Since, however, PL grids retrieved from MARS are irregularly spaced in the vertical, we need a method to map pressure to $t_{p}$. This is realized by means of an LUT stored in an additional 1-D texture. The level indices $k$ can be linearly scaled to $t_{p, k} \in(0 \ldots 1)$. Since we know the pressure values $p_{k}$ at the levels $k$, we can compute a continuous $\widetilde{k}$ for intermediate $p$ by linearly interpolating in $\ln (p)$ (Fig. 11b). $\widetilde{k}$ can subsequently be scaled to $t_{p}$. These mappings from $p$ to $t_{p}$ are pre-computed for a number, say 2048, of pressure values and stored in the LUT that can be accessed in the shader.

ML grids are not rectilinear and thus sampling becomes more complicated. As illustrated in Fig. 11b, the continuous level index $\widetilde{k}$ in general is not the same for adjacent grid columns. In the worst case, a given $p$ is located between different model levels in its four surrounding grid columns. Trilinear hardware interpolation requires $\widetilde{k}$ to be the same in all surrounding grid columns, it hence cannot be used. Consequently, we need to split the interpolation into four vertical interpolations in the grid columns and a subsequent bilinear horizontal interpolation. A naïve approach is to use the binary search used for the horizontal sections for the vertical interpolations; however, our experiments showed that rendering times can be reduced by a factor of about 2 when again making use of an LUT approach for hardware interpolation. However, the horizontal interpolation needs to be implemented in software. ML sampling is hence over 4 times more expensive than PL sampling. (a)

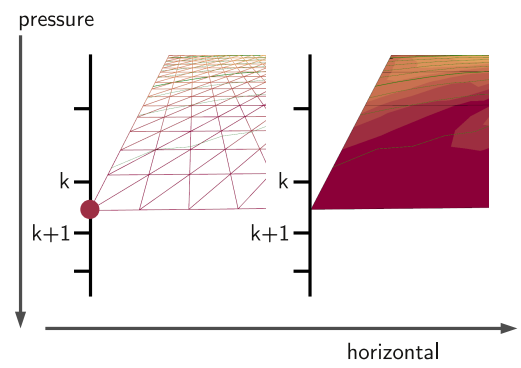

(b)

pressure

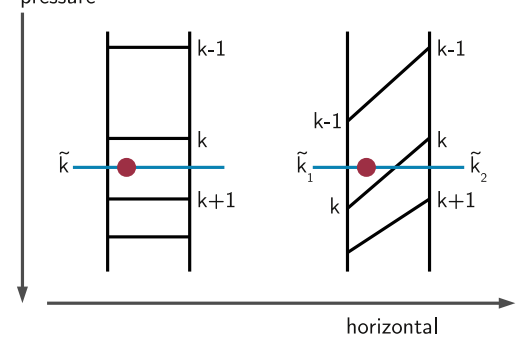

Figure 11. Sampling data fields in GPU shaders. (a) For each vertex of a horizontal section, model levels $k$ and $k+1$ are found by binary search. The scalar value is linearly interpolated in $\ln (p)$ between these two levels. (b) PL grids are rectilinear (left), allowing for the usage of trilinear hardware interpolation between the grid points surrounding a sample position (red dot). For ML grids (right), the sample position can be located between different model levels $k$ for two adjacent grid columns, thus prohibiting hardware interpolation.

To use hardware interpolation for the ML in the vertical, we need to extend the LUT approach. First, the horizontal texture coordinates $t_{\text {lon }}$ and $t_{\text {lat }}$ are set to the horizontal po- 
sition of the grid columns. Since the model level pressure varies with $p_{\text {sfc }}$, we in principle need to pre-compute one LUT for every $p_{\text {sfc }}$ value that occurs in the forecast field. We instead make use of a 2-D LUT, containing LUTs for discrete values of $p_{\text {sfc }}$ reflecting the expected range of $p_{\text {sfc }}$ in the data. Using bilinear hardware interpolation, this LUT is used to interpolate in both $p_{\text {sfc }}$ and $\ln (p)$ to obtain a mapping from $\ln (p)$ to $t_{p}$. The additional memory requirement is reasonable: for an LUT using 2048 entries in the vertical and 600 entries for $p_{\text {sfc }}$ between 1050 and $450 \mathrm{hPa}$, approximately $9 \mathrm{MB}$ of GPU memory are required in float precision (i.e. 4 bytes/value). The table can be shared among variables on the same grid.

The traversal of the data volume is accelerated with an empty-space skipping strategy (Krüger and Westermann, 2003). The longitude-latitude-pressure space covered by a data field is divided uniformly into a regular grid of $N_{i} \times$ $N_{j} \times N_{k}$ cells. For each cell, minimum and maximum data values are computed. In the shader, the information is used to skip cells in which an isosurface cannot possibly be located. Due to the different horizontal and vertical scales, care has to be taken when choosing the step size for traversing nonempty cells. Depending on the factor that is used to scale $\ln (p)$ to a $z$ coordinate in visualization space, the vertical distance between two grid points often is considerably smaller than the horizontal distance. The step size chosen needs to be small enough to ensure that no grid point is skipped during traversal.

Once an isosurface crossing has been identified, the isosurface normal (equivalent to the gradient of the scalar field at the crossing position) is computed via central differences. The pixel colour is subsequently determined using the commonly used Blinn-Phong lighting model (e.g Engel et al., 2006). Colour can be pre-defined or obtained from a transfer function. Also, a second scalar field can be mapped to the isosurface to colour, for example, a wind speed isosurface by temperature.

Table 2 lists typical rendering times for images shown in this paper. Note that the performance of the raycaster depends on the visualized data as well as on camera viewpoint. In particular the effectiveness of the empty-space skipping strategy for a selected isovalue depends strongly on the spatial distribution of the data values. During user interaction, the step size used by the raycaster to sample the data fields can be reduced (cf. Table 2). While this temporarily reduces image quality, rendering time is also reduced.

Two-dimensional sections are rendered at the same performance for ML and PL data sets, as the same number of interpolation operations needs to be performed for both grid types. For raycasted images, Table 2 provides timings for ML data sets and PL data sets with the same number of vertical levels. Due to the reduced number of vertical interpolation operations, PL data are typically rendered by a factor of two to three faster than ML data.
Table 2. Order of magnitude of rendering times achieved by Met.3D for selected visualizations from this paper. Timings are measured on the test hardware described in Sect. 3. ECMWF ENS data at a grid spacing of $1^{\circ}$ in both longitude and latitude are used. The data fields are available in GPU memory. ML refers to visualizations from hybrid sigma-pressure model levels (62 levels), PL refers to visualizations from data fields regridded to 62 pressure levels chosen equal to the levels of an ML grid defined by a constant surface pressure of $1000 \mathrm{hPa}$. Timings are average values of continuous rendering over $30 \mathrm{~s}$. A Met.3D window of 1600 by 900 pixels is used (the size used for the video in the Supplement, corresponding to a viewport of 1192 by 864 pixels). "Animated" for cross sections refers to vertically sliding a horizontal section or moving a waypoint of a vertical section.

\begin{tabular}{lccc}
\hline Figure & Setting & ML & PL \\
\hline Fig. 3b & static & \multicolumn{2}{c}{$2.3 \mathrm{~ms}$} \\
Fig. 3b & animated & \multicolumn{2}{c}{$2.8 \mathrm{~ms}$} \\
\hline Fig. 4a & static & \multicolumn{2}{c}{$6.2 \mathrm{~ms}$} \\
Fig. 4a & animated & \multicolumn{2}{c}{$6.8 \mathrm{~ms}$} \\
\hline Fig. 6a & step size 0.1 & $417 \mathrm{~ms}$ & $114 \mathrm{~ms}$ \\
Fig. 6a & step size 1 & $107 \mathrm{~ms}$ & $47 \mathrm{~ms}$ \\
\hline Fig. 7a & step size 0.1 & $222 \mathrm{~ms}$ & $73 \mathrm{~ms}$ \\
Fig. 7a & step size 1 & $62 \mathrm{~ms}$ & $39 \mathrm{~ms}$ \\
\hline Fig. 7c & step size 0.1 & $248 \mathrm{~ms}$ & $76 \mathrm{~ms}$ \\
Fig. 7c & step size 1 & $72 \mathrm{~ms}$ & $40 \mathrm{~ms}$ \\
\hline Fig. 2c & step size 0.1 & $273 \mathrm{~ms}$ & $100 \mathrm{~ms}$ \\
Fig. 2c & step size 1 & $83 \mathrm{~ms}$ & $67 \mathrm{~ms}$ \\
\hline
\end{tabular}

We note that as for the data pipeline, comprehensive optimizations of the visualization algorithms were outside the scope of our work. In particular with respect to the raycaster, further optimizations are possible, for example, by integrating an adaptive step size strategy.

\subsection{Computation of normal curves}

Normal curve computation is implemented in a compute shader. Figure 12 illustrates the proposed normal curve algorithm. To generate a set of seed points, rays aligned with the three world space axes (longitude, latitude, pressure) are cast through the data volume. The rays are started at regularly spaced points (grey arrows; the spacing can be adjusted by the user). To avoid the regular pattern of these initial start points being reflected by the normal curves, we disturb the ray positions by a random factor (black arrows). The intersection points of the rays with the selected outer isosurface are then used as initial seed points for the normal curves (green dots). In particular in regions of high curvature, multiple rays can hit the isosurface at close-by points on the surface. To prevent normal curves from being started close together, a regular volume with a grid size of the average initial ray distance is placed over the scene (yellow grid). Only one 


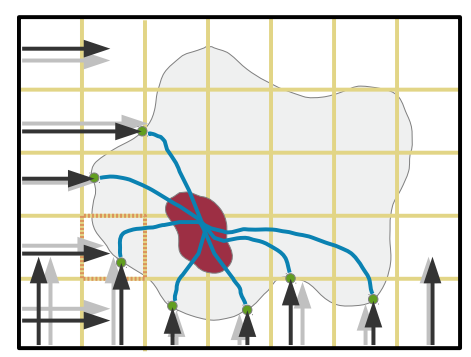

Figure 12. Computation of normal curves. Seeding points for the curves (green dots) are placed at the intersections between axis aligned rays (black arrows) and the outer isosurface (only rays from two directions are shown for illustration). Only a single seed is allowed in each grid box of the yellow volume.

seed is allowed per grid cell. Hence, if a seed point falls into a cell already occupied, it is discarded (illustrated in the orange grid cell). The normal curves are integrated in parallel in the direction of the scalar field's gradient, using a fourthorder Runge-Kutta scheme. The gradient is computed with the same method used for isosurface shading. If present, the integration can be stopped at an inner opaque isosurface (illustrated by the red isosurface in Fig. 12).

\section{Impact of (not) regridding on ensemble statistical quantities}

A challenge that arises from aiming at interactive ensemble visualization is the efficient yet accurate computation of statistical quantities from the ensemble predictions. We compute statistical quantities per grid point. Probabilities, for example, are computed by evaluating for every member and for each grid point a given probability criterion (for instance, wind speed exceeding a given threshold). The evaluation of the criterion yields for every member a binary volume, with the bits set when the criterion is fulfilled. Probabilities are computed by counting the number of members with a set bit for each grid point. Other statistical measures are computed similarly for each grid point over the ensemble dimension.

For 2-D grids, this is common procedure (Wilks, 2011) and also for 3-D grids not an issue as long as a given grid point is located at the same spatial position in all members. However, due to surface pressure varying between ensemble members, this is not the case for data on ML grids. Hence, depending on the vertical gradient of the forecast variable from which a statistical quantity is computed, an error is introduced. One approach to this issue is to vertically regrid all ensemble members to a common grid, for example, the one defined by the mean surface pressure (as done in the example pipeline in Fig. 10). This, however, introduces an additional interpolation step and demands computational resources.

In this section, we investigate the visual and quantitative differences between statistical quantities computed from the

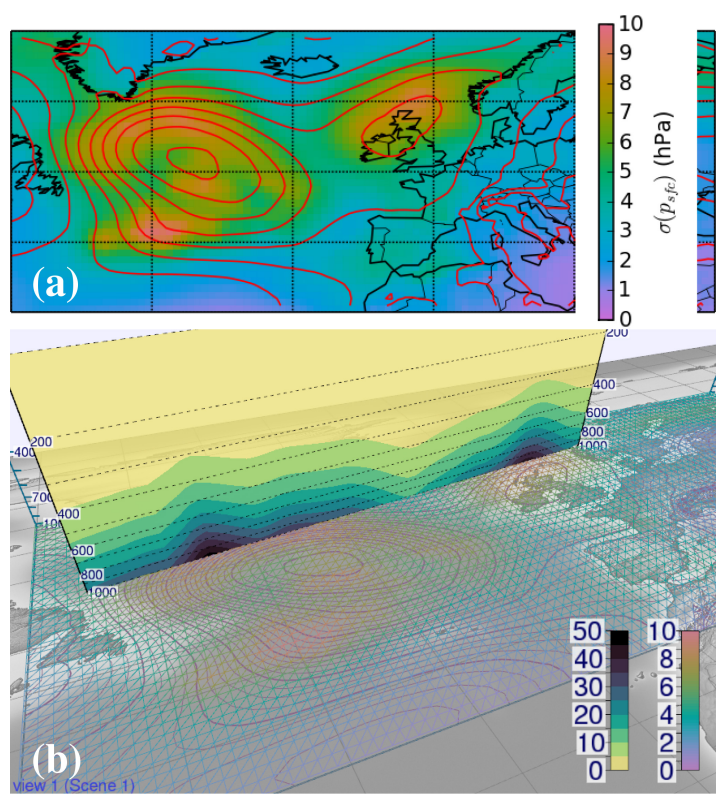

Figure 13. (a) $\mathrm{SD}$ of surface pressure, $\sigma\left(p_{\text {sfc }}\right)$. Forecast from 00:00 UTC, 15 October 2012, valid at 18:00 UTC, 19 October 2012. Red contour lines show mean sea level pressure. (b) Vertical section of the pressure difference (yellow-blue-black colour bar in $\mathrm{hPa}$ ) between highest and lowest ensemble member, rendered on top of a wireframe map of $\sigma\left(p_{\mathrm{sfc}}\right)$.

original ML grids and those computed from data fields regridded to a common grid. The differences are compared to an additional error that is introduced by linearly interpolating the statistical quantities. At ECMWF, maps of statistical quantities on pressure levels are computed from the individual member's forecast data on these pressure levels. This implies that a forecast meteorological variable is first interpolated to the target vertical position for each member (using linear interpolation in $p$ or $\ln (p)$; cf. Yessad, 2014), followed by the computation of the statistical quantity. If, on the contrary, we first compute the statistical quantity on the 3-D model grid and then linearly interpolate to the target vertical position, an error is introduced due to the non-linear nature of most statistical measures. The same problem arises in the horizontal dimensions.

In the following, we analyse regridding and interpolation error for the forecast data we had available from TNF. We present results from the forecast initialized at 00:00 UTC, 15 October 2012 and valid at $114 \mathrm{~h}$ lead time at 18:00 UTC, 19 October 2012. This case is representative for the data set, results for other time steps of the TNF data set are similar.

\subsection{Variation in grid-point pressure}

First, we estimate typical vertical grid-point displacements that can be observed between ensemble members. Figure 13a shows the SD of $p_{\text {sfc }}$ for the example case. It reaches values of 8 to $10 \mathrm{hPa}$ in the uncertain regions of the fore- 


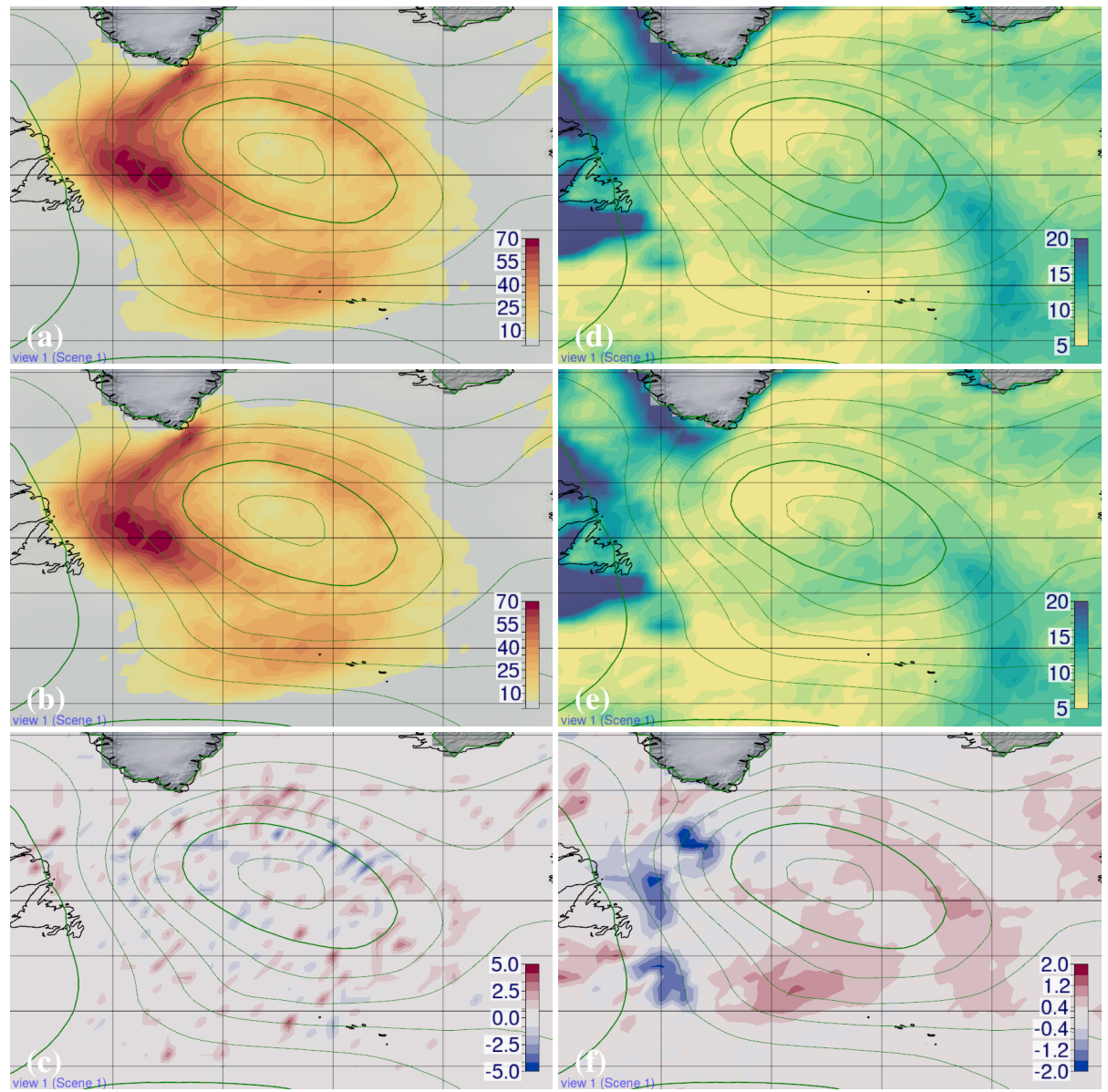

Figure 14. Visual differences between statistical quantities computed from a vertically regridded ensemble to those computed from the original ensemble. Horizontal section at $950 \mathrm{hPa}$ (approx. model levels 51-55 in Figs. 15 and 16) of (a-c) $p(|\boldsymbol{v}|>20 \mathrm{~m} \mathrm{~s}-1)(\%)$ and $(\mathbf{d}-$ f) $\sigma(\mathrm{RH})$. Same forecast as in Fig. 13. Shown is (a) the probability and (d) SD computed from the original model grid, (b and e) computed from members regridded to the grid defined by the mean $p_{\text {sfc }}$, and (c and $\left.\mathbf{f}\right)$ the difference between both fields.

cast. This particularly applies to the low-pressure systems over the Atlantic and the northern British Isles. Figure 13b shows a vertical cross section of the maximum pressure difference between any two members per grid point in these two areas. Close to the surface, the difference reaches $40 \mathrm{hPa}$, corresponding (at low altitudes) to an elevation offset of about $400 \mathrm{~m}$. In most other regions, however, differences are smaller. Also, as expected from the model grid topology, differences vanish in upper atmospheric levels.

\subsection{Difference due to vertical regridding}

Vertical regridding is implemented as a data source that can be integrated into the Met.3D ensemble processing pipeline (cf. Fig. 10). The user can toggle between visualizations from original and from regridded data fields, and, if required, permanently enable regridding. If statistical quantities are computed from the original member grids, the resulting field is interpreted on a grid defined by the mean surface pressure.
On our test hardware (cf. Sect. 3), the cost of singlethreaded CPU regridding on average is about $60 \mathrm{~ms}$ per member and variable for the TNF ENS forecast at $1^{\circ}$ grid spacing (256742 grid points per 3-D field) and about $1 \mathrm{~s}$ at $0.25^{\circ}$ grid spacing (4997262 grid points). Even though multiple ensemble members can be processed in parallel on a multi-core machine and the regridding process could be further sped up using the GPU, there is a delay in particular for highresolution data sets and visualizations using multiple variables.

We have visually inspected a number of 2-D and 3-D renderings of statistical quantities of several meteorological variables. As expected, the largest visual differences appear close to the surface. They become most manifest in horizontal sections, which are most sensitive to vertical variations in a 3-D data field. Figure 14 shows two typical low-altitude examples, the probability of horizontal wind speed exceeding $20 \mathrm{~m} \mathrm{~s}^{-1}, p\left(|\boldsymbol{v}|>20 \mathrm{~m} \mathrm{~s}^{-1}\right)$, and the SD of relative humidity, $\sigma(\mathrm{RH})$. From our inspection we find that differences 

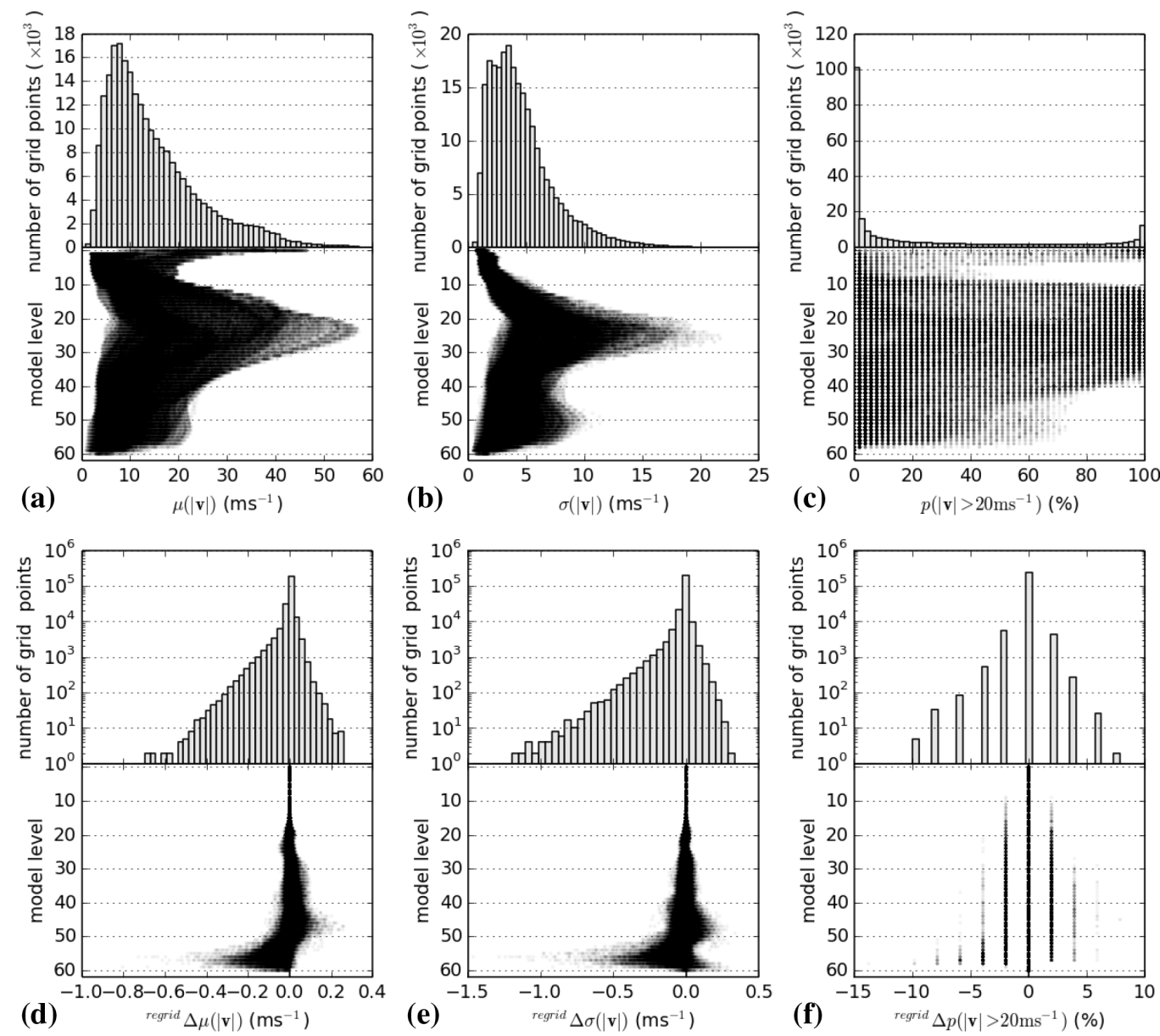

Figure 15. Distribution of differences between statistical quantities computed from a vertically regridded ensemble to those computed from the original ensemble. Plots are generated from all 256742 grid points of the data field. Same forecast as in Fig. 13. Shown are (a and d) $\mu(|\boldsymbol{v}|),(\mathbf{b}$ and $\mathbf{e}) \sigma(|\boldsymbol{v}|)$ and $(\mathbf{c}$ and $\mathbf{f}) p\left(|\boldsymbol{v}|>20 \mathrm{~ms}^{-1}\right) ;(\mathbf{a}-\mathbf{c})$ distribution and vertical occurrence of absolute values of the quantities. (d-f) Distribution and vertical occurrence of differences due to regridding (denoted by ${ }^{\text {regrid }} \Delta$ ); note the logarithmic scale of the histograms in (d-f). Probability values are discrete due to the size of the ensemble (51 members).

tend to be larger for variables that depend on moisture and variables derived thereof; however, we could not find any examples in which visualized structures were significantly altered. For example, while there is some visible difference in $\sigma(\mathrm{RH})$ along Rafael's warm front, the structure itself is not significantly altered.

Visual differences strongly depend on the employed colour palette and visualized data range. Depending on the range of values covered by a single colour, small changes might simply not be reflected in the visualization. To ensure that differences in general are small, we have performed a statistical analysis of the entire TNF data set. Figure 15 shows results for three statistical quantities computed from the wind field of the example case: mean $\mu(|\boldsymbol{v}|)$, SD $\sigma(|\boldsymbol{v}|)$, and $p\left(|\boldsymbol{v}|>20 \mathrm{~m} \mathrm{~s}^{-1}\right)$. The scatter plots show that for all three quantities the largest differences appear at lower altitudes (higher model level indices). Also, differences mostly are small compared to absolute values of the quantities. For ex- ample, at only a few grid points the difference in $\sigma(|\boldsymbol{v}|)$ and $p\left(|\boldsymbol{v}|>20 \mathrm{~m} \mathrm{~s}^{-1}\right)$ exceeds $1 \mathrm{~m} \mathrm{~s}^{-1}$ and $10 \%$, respectively. The range of differences observed in Fig. 14 is well reflected in the histogram.

Larger differences appear for statistical quantities computed from moist variables (Fig. 16). Again, the histogram for $\sigma(\mathrm{RH})$ confirms the range of differences shown in Fig. 14 (Fig. 16d). For probabilities of potential vorticity and cloud cover, differences of up to $30 \%$ can occur (Fig. 16e and f). However, for most grid points, differences are smaller.

Figure 17 shows a histogram of $\sigma\left(p_{\text {sfc }}\right)$ of the example case, overlain with the bin-averaged difference in $\sigma(|\boldsymbol{v}|)$. As can be expected, larger differences on average occur in regions with high $\sigma\left(p_{\text {sfc }}\right)$. However, even for large $\sigma\left(p_{\text {sfc }}\right)$, most differences are small (not shown). We hence cannot state that large $\sigma\left(p_{\text {sfc }}\right)$ in general accounts for large differences. 

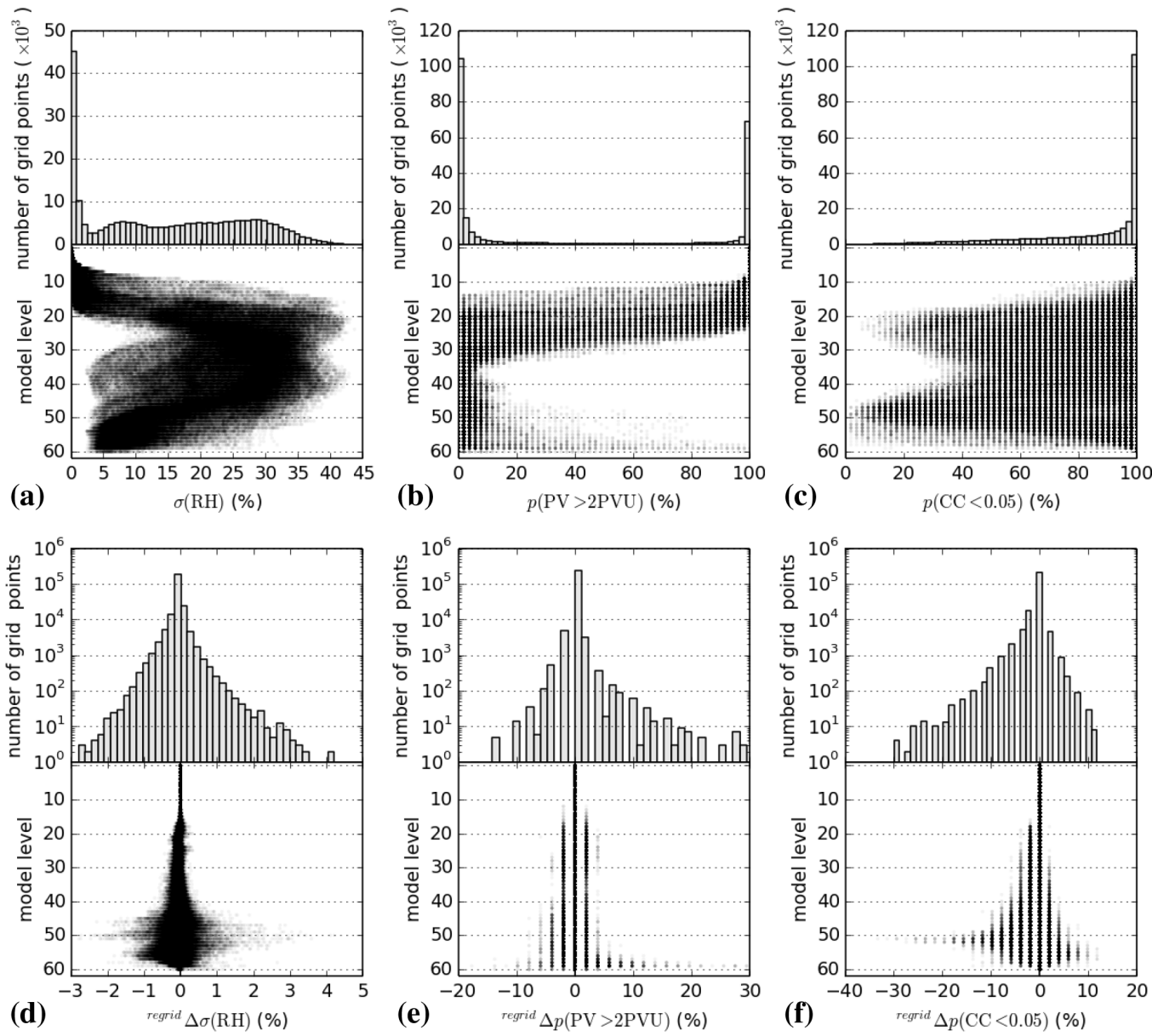

Figure 16. The same as Fig. 15 but for variables depending on moisture; (a and d) SD of relative humidity; (b and e) probability of potential vorticity exceeding 2 PVU; (c and f) Probability of grid box cloud cover fraction falling below 0.05 .

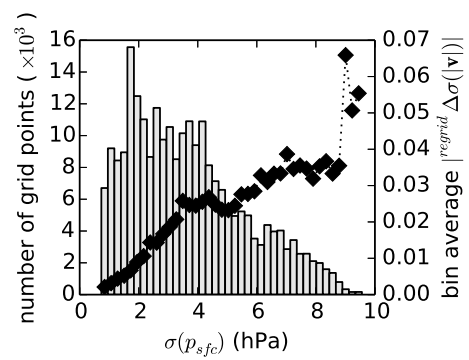

Figure 17. Histogram of $\sigma\left(p_{\mathrm{sfc}}\right)$, overlain with the bin-averaged difference of $\sigma\left(p_{\text {sfc }}\right)$ against the differences between $\sigma(|\boldsymbol{v}|)$ computed from a vertically regridded ensemble and computed from the original member grids. Same forecast as in Fig. 13.

\subsection{Error due to vertical interpolation of statistical quantities}

The error introduced by vertical linear interpolation of a statistical quantity depends on the quantity. Consider the example given in Table 3. Due to the linear nature of the ensemble mean, there is no difference whether we first compute the mean at the grid points and then interpolate to the sample location or vice versa. For non-linear quantities including SD and probability, the results are different.

Figure 18 shows distributions of the interpolation errors for $\sigma(|\boldsymbol{v}|)$ and $p\left(|\boldsymbol{v}|>20 \mathrm{~m} \mathrm{~s}^{-1}\right)$. Note that in contrast to the differences caused by regridding, the largest errors due to interpolation occur in upper atmospheric levels, where the vertical distance between model levels becomes larger. Between the surface and approximately model level 10 (approximately $100 \mathrm{hPa}$ ), the order of magnitude of the interpolation errors is comparable to that of the differences due to regridding. At middle atmospheric levels, both errors are at a minimum, as shown by the vertical profile of horizontally averaged differences. At the upper boundary of the model atmosphere, interpolation errors become significantly larger, These regions, however, are not relevant for the forecast cases we are interested in. 

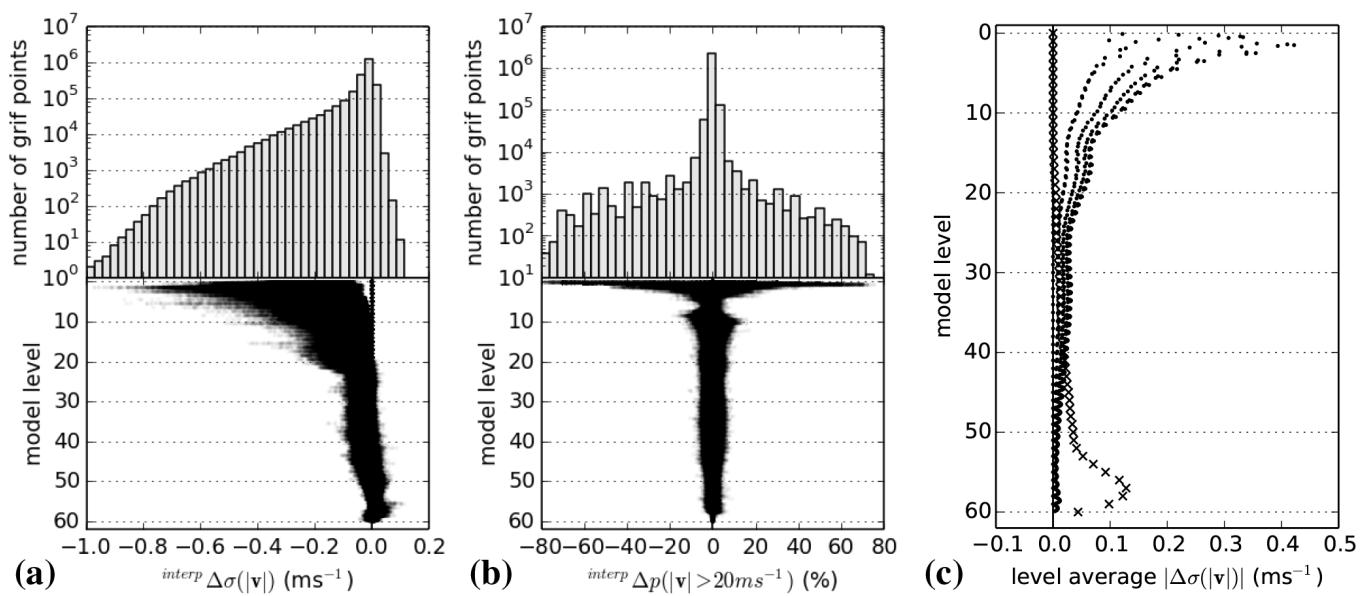

Figure 18. Distribution of errors due to vertical linear interpolation (denoted by interp $\Delta$ ) of statistical quantities. (a) Distribution of errors of $\sigma(|\boldsymbol{v}|)$ (top), and vertical occurrence of the errors (bottom). (b) The same for $p\left(|\boldsymbol{v}|>20 \mathrm{~ms}^{-1}\right.$ ). (c) Vertical profile of level average differences due to regridding (crosses) and interpolation (dots). Same forecast as in Fig. 13.

Table 3. Example of vertically interpolating statistical quantities. Consider an ensemble of three members and corresponding scalar quantities $s_{1} \ldots s_{3}$ at the two vertical levels $k$ and $k+1$. While the mean value $\mu(s)$, interpolated to the mid-level between $k$ and $k+1$, equals the mean of the interpolated scalar values, this is not true for the $\operatorname{SD} \sigma(s)$ and the probability that a scalar value exceeds $1.5, p(s>1.5)$. The subscript i refers to "interpolated".

\begin{tabular}{lccccccccc}
\hline Level & $s_{1}$ & $s_{2}$ & $s_{3}$ & $\mu\left(s_{\mathrm{i}}\right)$ & $\mu_{\mathrm{i}}(s)$ & $\sigma\left(s_{\mathrm{i}}\right)$ & $\sigma_{\mathrm{i}}(s)$ & $p\left(s_{\mathrm{i}}>1.5\right)$ & $p_{\mathrm{i}}(s>1.5)$ \\
\hline$k$ & 0.8 & 1.7 & 1.8 & 1.433 & & 0.45 & & 0.66 & \\
Mid-level & 1.4 & 1.45 & 1.4 & 1.4166 & 1.4166 & 0.24 & 0.44 & 0 & 0.5 \\
$k+1$ & 2.0 & 1.2 & 1.0 & 1.40 & & 0.43 & & 0.33 & \\
\hline
\end{tabular}

\subsection{Discussion}

The examples show that the errors introduced by computing the statistical quantities from the original member grids are of comparable magnitude to the errors introduced by vertically interpolating the computed quantities. For most grid points, both are negligible and result in only little difference in the visualization. However, for some variables and cases (in particular moist variables), differences can be of the same order of magnitude as the statistical quantity itself.

We conclude that for general exploration of the forecast data, it is sufficient for the user to use the "fast" option and visualize quantities computed from the original member grids. However, if the result is crucial for an important decision, our advice is to switch to regridded quantities and accept the additional compute time. The "best" results and those most comparable to products obtained from ECMWF can be achieved by first interpolating each member to the desired vertical pressure and then computing the statistical quantities. In this case, neither regridding nor vertical interpolation of the quantity corrupts the result. In Met.3D, this is possible for horizontal sections.

\section{Conclusions}

We have presented Met.3D, a new open-source tool that provides interactive 3-D visualization techniques for numerical ensemble weather prediction data in a way suitable for weather forecasting. The development of Met.3D has been motivated by the application of forecasting during aircraft-based atmospheric field campaigns, in particular, by the requirements of the T-NAWDEX-Falcon 2012 campaign. However, we see the tool applicable to a wider range of applications, including the analysis of ensemble simulation output in atmospheric research and the usage of Met.3D to support teaching in meteorology classes.

Our work is concerned with meaningful 3-D depiction and ensemble visualization, two challenging topics of weather forecast visualization. We have addressed a number of challenges that have been discussed in the literature, including prevention of a decoupling between commonly used 2-D and new 3-D visualization methods, spatial perception in 3-D scenes, suitable uncertainty visualization techniques, and system performance. Interactivity is key to our approach. It is facilitated by exploiting the computational power provided by modern graphics processing units and by means of a flexible, modular system architecture. We have built a bridge from 
proven 2-D visualization methods commonly used in meteorology to 3-D visualization; 2-D products are rendered in a 3-D context, a product's position can be changed interactively. When 3-D elements are visualized, spatial perception is improved by displaying shadows on the Earth's surface, enabling the user to judge the horizontal position and relative elevation of an element. Quantitative height information can be obtained by means of interactive vertical axes. We have proposed normal curves, a novel visualization technique to reveal the structure inside a transparent 3-D isosurface of a scalar field. With normal curves, the locations and magnitudes of local extrema in the visualized data can be identified at a glance. To visually provide information on forecast uncertainty, Met.3D implements support for ensemble forecasts. The tool is designed to allow for integration of both feature-based and location-based ensemble visualization techniques. In the presented version, forecast products can be animated over the ensemble dimension, and statistical quantities can be derived and visualized on demand. Concerning the computation of statistical quantities from forecast data on hybrid sigma-pressure grids, we have shown that ignoring the variation in grid-point pressure between the ensemble members has little impact on the visualization.

The paper at hand is the first of a two-part study. We have focussed on Met.3D's functionality, system architecture and visualization algorithms. In Part 2, we focus on the specific forecast requirements of T-NAWDEX-Falcon and use Met.3D to predict warm conveyor belt situations. Ensemble particle trajectories are employed to predict a probability of warm conveyor belt occurrence. In particular, a case study, revisiting a forecast case from T-NAWDEX-Falcon, demonstrates the practical application of Met.3D and highlights the potential of the software to improve the weather forecasting process.

Future work needs to include careful evaluation of the presented visualization techniques to study their impact on tasks performed by meteorologists and atmospheric researchers in their daily work. We discuss our point of view on the added value of interactive 3-D ensemble visualization for forecasting after the presentation of the case study in the conclusions of Part 2. For example, in our experience, the provided interactivity for 2-D sections and the ability to add features as 3-D elements helps to much faster build a mental model of the atmosphere. This, of course, reflects our personal perception. We plan to evaluate the issue with a user study in the near future.

We will actively use Met.3D during upcoming field campaigns, including a future NAWDEX campaign scheduled for 2016. We also see much potential for further research in meteorological visualization. With respect to $3-\mathrm{D}$ visualization, further improvement of spatial perception is very important. In the Met.3D version presented here, shadows are only rendered on the Earth's surface. Global illumination techniques (e.g. Jönsson et al., 2014) that, for example, allow 3-D elements to mutually cast shadows on each other, may fur- ther improve the user's judgement of spatial relationships. Also, the impact of different projections on perceived spatial distance needs to be studied. Met.3D currently is restricted to a cylindrical map projection in the horizontal. Additional challenges include the efficient rendering from further native model grid topologies and real-time placement of text labels to convey quantitative information. The latter applies in particular to 2-D and 3-D contour lines and surfaces. Due to the employed GPU implementation of the 2-D marching squares contouring algorithm, continuous line geometry is not easily available. Hence, it is difficult to compute positions for labels.

With respect to ensemble and uncertainty visualization, open questions are abundant, as reflected by the literature surveyed in Sect. 2. In Part 2, we introduce a feature-based approach for WCBs. Further approaches, both feature based and location based, can be implemented in Met.3D to study their feasibility and applicability in meteorology.

With the development of Met.3D, we have demonstrated how we envision 3-D and ensemble techniques to become a part of standard meteorological visualization. The tool provides a solid software infrastructure that opens the door to investigate the above-listed and other research questions, thus enabling the further advancement of meteorological visualization.

\section{Code availability}

To facilitate ease of deployment and of future research and developments, we have made the source code of Met.3D available as open-source under the GNU General Public License, version 3. Please enter the following into your web browser to go to the software repository: https://bitbucket. org/wxmetvis/met.3d; here you can obtain an up-to-date version of the software. We welcome user feedback as well as contributions that help with the further development of the code. If you are interested, please contact us.

\section{The Supplement related to this article is available online at doi:10.5194/gmd-8-2329-2015-supplement.}

Acknowledgements. Access to ECMWF prediction data has been kindly provided in the context of the ECMWF special project "Support Tool for HALO Missions". This work was supported by the European Union under the ERC Advanced Grant 291372 SaferVis - Uncertainty Visualization for Reliable Data Discovery. M. Rautenhaus was supported by a grant from Ev. Studienwerk Villigst e.V. C. M. Grams and A. Schäfler were supported by the German Research Foundation (DFG) as part of the research unit PANDOWAE (FOR896).

This work was supported by the German Research Foundation (DFG) and the Technische Universität 
München within the funding programme

Open Access Publishing.

Edited by: H. Tost

\section{References}

Abram, G. and Treinish, L.: An Extended Data-Flow Architecture for Data Analysis and Visualization, in: Proceedings of the 6th Conference on Visualization '95, VIS '95, IEEE Computer Society, Washington, DC, USA, 1995.

Alpert, J. C.: 3-dimensional animated displays for sifting out medium range weather events, in: 19th Conference on IIPS, 9-13 February 2003, Long Beach, California, 2003.

Bailey, M.: Using GPU shaders for visualization, IEEE Comput. Graph., 29, 96-100, 2009.

Bailey, M.: Using GPU shaders for visualization, Part 2, IEEE Comput. Graph., 31, 67-73, 2011.

Bailey, M.: Using GPU shaders for visualization, Part 3, IEEE Comput. Graph., 33, 5-11, 2013.

Barjenbruch, D. B., Thaler, E., and Szoke, E. J.: Operational Applications of Three Dimensional Air Parcel Trajectories Using AWIPS D3D, in: Proceedings of the 18th IIPS Conference, 1318 January 2002, Orlando, Florida, 2002.

Böttinger, M., Gülzow, V., and Biercamp, J.: Visualisierung als Werkzeug zur Analyse von Klimasimulationsdaten, in: Umweltinformatik '98: Vernetzte Strukturen in Informatik, Umwelt und Wirtschaft, edited by: Haasis, H. D. and Ranze, K. C., 1998.

Böttinger, M., Meier-Fleischer, K., and Ulmen, C.: Tutorial: Interactive 3D Visualization in Earth System Research with Avizo Green 8.0, DKRZ/KlimaCampus Hamburg, 2013.

Brown, R.: Animated visual vibrations as an uncertainty visualisation technique, in: Proceedings of the 2nd International Conference on Computer Graphics and Interactive Techniques in Australasia and South East Asia, GRAPHITE '04, ACM, New York, NY, USA, 84-89, 2004.

Browning, K. A. and Roberts, N. M.: Structure of a frontal cyclone, Q. J. Roy. Meteor. Soc., 120, 1535-1557, 1994.

Buizza, R., Bidlot, J.-R., Wedi, N., Fuentes, M., Hamrud, M., Holt, G., Palmer, T., and Vitart, F.: The ECMWF Variable Resolution Ensemble Prediction System VAREPS, ECMWF Newsletter, 108, 14-20, 2006.

Clyne, J., Mininni, P., Norton, A., and Rast, M.: Interactive desktop analysis of high resolution simulations: application to turbulent plume dynamics and current sheet formation, New J. Phys., 9, 301, doi:10.1088/1367-2630/9/8/301, 2007.

Cox, J., House, D., and Lindell, M.: Visualizing uncertainty in predicted hurricane tracks, Int. J. Uncertainty Quant., 3, 143-156, 2013.

Djurcilov, S., Kim, K., Lermusiaux, P., and Pang, A.: Visualizing scalar volumetric data with uncertainty, Comput. Graph., 26, 239-248, 2002.

Ducrocq, V., Braud, I., Davolio, S., Ferretti, R., Flamant, C., Jansa, A., Kalthoff, N., Richard, E., Taupier-Letage, I., Ayral, P.-A., Belamari, S., Berne, A., Borga, M., Boudevillain, B., Bock, O., Boichard, J.-L., Bouin, M.-N., Bousquet, O., Bouvier, C., Chiggiato, J., Cimini, D., Corsmeier, U., Coppola, L., Cocquerez, P., Defer, E., Delanoë, J., Di Girolamo, P., Doerenbecher, A.,
Drobinski, P., Dufournet, Y., Fourrié, N., Gourley, J. J., Labatut, L., Lambert, D., Le Coz, J., Marzano, F. S., Molinié, G., Montani, A., Nord, G., Nuret, M., Ramage, K., Rison, W., Roussot, O., Said, F., Schwarzenboeck, A., Testor, P., Van Baelen, J., Vincendon, B., Aran, M., and Tamayo, J.: HyMeX-SOP1: The Field Campaign Dedicated to Heavy Precipitation and Flash Flooding in the Northwestern Mediterranean, Bull. Amer. Meteor. Soc., 95, 1083-1100, 2014.

Dyer, J. and Amburn, P.: Desktop visualization of meteorological data using paraview, Kitware Source, 14, 7-10, 2010.

Elsberry, R. L. and Harr, P. A.: Tropical cyclone structure (TCS08) field experiment science basis, observational platforms, and strategy, Asia-Pacific J. Atmos. Sci., 44, 209-231, 2008.

Engel, K., Hadwiger, M., Kniss, J., Rezk-Salama, C., and Weiskopf, D.: Real-Time Volume Graphics, 1st Edn., AK Peters, Wellesley, Mass, 2006.

Favre, J. M. and Valle, M.: AVS and AVS/Express, in: The Visualization Handbook, edited by Hansen, C. D. and Johnson, C., Chap. 33, 655-672, Academic Press, 2005.

Gallus, W. A., Yarger, D. N., Cruz-Neira, C., and Heer, R.: An Example of a Virtual Reality Learning Environment, Bull. Amer. Meteor. Soc., 84, 18-20, 2003.

Gallus, W. A., Cervato, C., Cruz-Neira, C., Faidley, G., and Heer, R.: Learning Storm Dynamics with a Virtual Thunderstorm, Bull. Amer. Meteor. Soc., 86, 162-163, 2005.

Gneiting, T. and Raftery, A. E.: Weather forecasting with ensemble methods, Science, 310, 248-249, 2005.

Grigoryan, G. and Rheingans, P.: Point-based probabilistic surfaces to show surface uncertainty, IEEE T. Vis. Comput. Gr., 10, 564 573, 2004.

Grotjahn, R. and Chervin, R. M.: Animated Graphics in Meteorological Research and Presentations, Bull. Amer. Meteor. Soc., 65, 1201-1208, 1984.

Hansen, C. D. and Johnson, C.: The Visualization Handbook, 1st Edn., Academic Press, Burlington, MA, 2005.

Heizenrieder, D. and Haucke, S.: Das meteorologische Visualisierungs- und Produktionssystem NinJo, promet, 35, 57-69, 2009.

Henderson, A., Ahrens, J., and Law, C.: The ParaView Guide, Kitware, Clifton Park, NY, 2004.

Hewson, T. D. and Titley, H. A.: Objective identification, typing and tracking of the complete life-cycles of cyclonic features at high spatial resolution, Met. Apps, 17, 355-381, 2010.

Hibbard, W. L.: Computer-Generated Imagery for 4-D Meteorological Data, Bull. Amer. Meteor. Soc., 67, 1362-1369, 1986.

Hibbard, W. L.: VisAD: Connecting People to Computations and People to People, SIGGRAPH Comput. Graph., 32, 10-12, 1998.

Hibbard, W. L.: The top five problems that motivated my work, IEEE Comput. Graph., 24, 9-13, 2004.

Hibbard, W. L.: Vis5D, Cave5D, and VisAD, in: The Visualization Handbook, edited by Hansen, C. D. and Johnson, C., chap. 34, pp. 673-688, Academic Press, 2005.

Hibbard, W. L. and Santek, D.: The VIS-5D system for easy interactive visualization, in: Proceedings of the 1st Conference on Visualization '90, VIS '90, San Francisco, CA, USA, 23-26 October 1990, IEEE Computer Society Press, Los Alamitos, CA, USA, 28-35, 1990.

Hibbard, W. L., Santek, D., Uccellini, L., and Brill, K.: Application of the 4-D McIDAS to a Model Diagnostic Study of the Presi- 
dents' Day Cyclone, Bull. Amer. Meteor. Soc., 70, 1394-1403, 1989.

Hoffman, R. R. and Coffey, J. W.: Weather forecasting and the principles of complex cognitive systems, in: Proceedings of the Human Factors and Ergonomics Society Annual Meeting, Vol. 48, New Orleans, Louisiana, 20-24 September 2004, SAGE Publications, 315-319, doi:10.1177/154193120404800309, 2004.

Hoffman, R. R., Detweiler, M., Conway, J. A., and Lipton, K.: Some considerations in using color in meteorological displays, Weather Forecast., 8, 505-518, 1993.

Höllt, T., Magdy, A., Zhan, P., Chen, G., Gopalakrishnan, G., Hoteit, I., Hansen, C. D., and Hadwiger, M.: Ovis: a framework for visual analysis of ocean forecast ensembles, IEEE T. Vis. Comput. Gr., 20, 1114-1126, doi:10.1109/TVCG.2014.2307892, 2014.

Johnson, C. R. and Sanderson, A. R.: A next step: visualizing errors and uncertainty, IEEE Comput. Graph., 23, 6-10, 2003.

Jönsson, D., Sundén, E., Ynnerman, A., and Ropinski, T.: A survey of volumetric illumination techniques for interactive volume rendering, Comput. Graphics Forum, 33, 27-51, 2014.

Kaufmann, S., Voigt, C., Jeßberger, P., Jurkat, T., Schlager, H., Schwarzenboeck, A., Klingebiel, M., and Thornberry, T.: In situ measurements of ice saturation in young contrails, Geophys. Res. Lett., 41, 702-709, doi:10.1002/2013g1058276, 2014.

Koppert, H. J., Schröder, F., Hergenröther, E., Lux, M., and Trembilski, A.: 3-D visualisation in daily operation at the DWD, in: Proceedings of the 6th ECMWF Workshop on Meteorological Operational Systems, Reading, England, 17-21 November 1997, 119-142, 1998.

Krüger, J. and Westermann, R.: Acceleration techniques for GPUbased volume rendering, in: Proceedings of the 14th IEEE Visualization 2003 (VIS'03), Seattle, Washington, 22-24 October 2003, 287-292, IEEE Computer Society, Washington, DC, USA, doi:10.1109/VIS.2003.10001, 2003.

Lalaurette, F.: Early detection of abnormal weather conditions using a probabilistic extreme forecast index, Q. J. Roy. Meteor. Soc., 129, 3037-3057, 2003.

Lamy-Thépaut, S., Sahin, C., and Raoult, B.: ecCharts service, ECMWF Newsletter, 134, 7-9, 2013.

Leutbecher, M. and Palmer, T.: Ensemble forecasting, J. Comput. Phys., 227, 3515-3539, 2008.

Lindemann, F. and Ropinski, T.: About the influence of illumination models on image comprehension in direct volume rendering, IEEE T. Vis. Comput. Gr., 17, 1922-1931, 2011.

Lundstrom, C., Ljung, P., Persson, A., and Ynnerman, A.: Uncertainty visualization in medical volume rendering using probabilistic animation, IEEE T. Vis. Comput. Gr., 13, 1648-1655, 2007.

Lux, M., and Frühauf, T.: A visualization system for operational meteorological use, in: Proceedings of the Sixth International Conference in Central Europe on Computer Graphics and Visualization (WSCG'98), Plzen, Czech Republic, 9-13 February 1998, 525-534, 1998.

McCaslin, P. T., McDonald, P. A., and Szoke, E. J.: 3-D visualization development at NOAA forecast systems laboratory, Comp. Graph., 34, 41-44, 2000.

Middleton, D., Scheitlin, T., and Wilhelmson, B.: Visualization in weather and climatic research, in: The Visualization Handbook,
Chap. 44, edited by: Hansen, C. D. and Johnson, C., Academic Press, 845-871, 2005.

Miller, M., Buizza, R., Haseler, J., Hortal, M., Janssen, P., and Untch, A.: Increased resolution in the ECMWF deterministic and ensemble prediction systems, ECMWF Newsletter, 124, 10-16, 2010.

Murray, D. and McWhirter, J.: Evolving IDV - creating better tools for the community, in: 23th Conference on International Interactive Information and Processing Systems (IIPS) for Meteorology, Oceanography, and Hydrology, 15-18 January 2007, San Antonio, TX, American Meteorological Society, 3B.5, 2007.

Murray, D., McWhirter, J., Ho, Y., and Whittaker, T. M.: IDV at 5: new features and future, in: 25th Conference on International Interactive Information and Processing Systems (IIPS) for Meteorology, Oceanography, and Hydrology, 10-15 January 2009, Phoenix, AZ, American Meteorological Society, 7B.5, 2009.

Nietfeld, D. D.: The Synoptic Environment of the 11 April 2001 Central Plains Tornado Outbreak Viewed in Three Dimensions, in: Proceedings of the 19th IIPS Conference, 9-13 February 2003, Long Beach, California, 2003.

Nietfeld, D. D.: The utility of three-dimensional radar displays in severe weather warning operations, in: 23rd Conference on Severe Local Storms, 5-11 November 2006, St. Louis, MO, 2006.

Norton, A. and Clyne, J.: The VAPOR visualization application, in: High Performance Visualization, Chap. 20, edited by: Bethel, E. W., Childs, H., and Hansen, C., CRC Press, Boca Raton, FL, 415-428, 2012.

Obermaier, H. and Joy, K. I.: Future challenges for ensemble visualization, IEEE Comput. Graph., 34, 8-11, 2014.

Pang, A. T., Wittenbrink, C. M., and Lodha, S. K.: Approaches to uncertainty visualization, Visual Comput., 13, 370-390, 1997.

Papathomas, T. V., Schiavone, J. A., and Julesz, B.: Applications of Computer Graphics to the Visualization of Meteorological Data, SIGGRAPH Comput. Graph., 22, 327-334, 1988.

Pfaffelmoser, T. and Westermann, R.: Visualization of global correlation structures in uncertain 2-D scalar fields, Comput. Graph. Forum, 31, 1025-1034, 2012.

Pfaffelmoser, T., Reitinger, M., and Westermann, R.: Visualizing the positional and geometrical variability of isosurfaces in uncertain scalar fields, Comput. Graph. Forum, 30, 951-960, 2011.

Pöthkow, K. and Hege, H. C.: Positional uncertainty of isocontours: condition analysis and probabilistic measures, IEEE T. Vis. Comput. Gr., 17, 1393-1406, doi:10.1109/tvcg.2010.247, 2011.

Pöthkow, K., Weber, B., and Hege, H.-C.: Probabilistic marching cubes, Comput. Graph. Forum, 30, 931-940, 2011.

Potter, K., Wilson, A., Bremer, P. T., Williams, D., Doutriaux, C., Pascucci, V., and Johnson, C. R.: Ensemble-Vis: a framework for the statistical visualization of ensemble data, in: Int. Conference on Data Mining Workshops, Miami, FL, 6 December 2009, IEEE Computer Society, Los Alamitos, CA, USA, 233 240, doi:10.1109/ICDMW.2009.55, 2009.

Rautenhaus, M., Bauer, G., and Dörnbrack, A.: A web service based tool to plan atmospheric research flights, Geosci. Model Dev., 5, 55-71, doi:10.5194/gmd-5-55-2012, 2012.

Rautenhaus, M., Grams, C. M., Schäfler, A., and Westermann, R.: GPU based interactive 3-D visualization of ECMWF ensemble forecasts, ECMWF Newsletter, 138, 34-38, 2014.

Rautenhaus, M., Grams, C. M., Schäfler, A., and Westermann, R.: Three-dimensional visualization of ensemble weather forecasts 
- Part 2: Forecasting warm conveyor belt situations for aircraftbased field campaigns, Geosci. Model Dev., 8, 2355-2377, doi:10.5194/gmd-8-2355-2015, 2015.

Rhodes, P. J., Laramee, R. S., Bergeron, R. D., and Sparr, T. M.: Uncertainty visualization methods in isosurface rendering, in: Proceedings Eurographics 2003, Granada, Spain, 1-5 September 2003, 83-88, 2003.

Russell, I., Siemen, S., Ii, F., Kertész, S., Lamy-Thépaut, S., and Karhila, V.: Metview 4 - ECMWF's latest generation meteorological workstation, ECMWF Newsletter, 126, 23-27, 2010.

Sanyal, J., Zhang, S., Dyer, J., Mercer, A., Amburn, P., and Moorhead, R.: Noodles: a tool for visualization of numerical weather model ensemble uncertainty, IEEE T. Vis. Comput. Gr., 16, 1421-1430, 2010.

Schäfler, A., Boettcher, M., Grams, C. M., Rautenhaus, M., Sodemann, H., and Wernli, H.: Planning aircraft measurements within a warm conveyor belt, Weather, 69, 161-166, 2014.

Schiavone, J. A. and Papathomas, T. V.: Visualizing Meteorological Data, Bull. Amer. Meteor. Soc., 71, 1012-1020, 1990.

Schröder, F.: Visualisierung meteorologischer Daten, Springer, Berlin, Heidelberg, 1997.

Schumann, U.: On conditions for contrail formation from aircraft exhausts, Meteorol. Z., 5, 4-23, 1996.

Stalling, D., Westerhoff, M., and Hege, H.-C.: amira: A Highly Interactive System for Visual Data Analysis, in: The Visualization Handbook, edited by Hansen, C. D. and Johnson, C., chap. 38, 749-767, Academic Press, 2005.

Stauffer, R., Mayr, G. J., Dabernig, M., and Zeileis, A.: Somewhere over the rainbow: How to make effective use of colors in meteorological visualizations, Bull. Amer. Meteor. Soc., 96, 203-216, doi:10.1175/bams-d-13-00155.1, 2015.

Szoke, E. J., Grote, U. H., McCaslin, P. T., and McDonald, P. A.: D3D update: is it being used?, in: Proceedings of the 19th IIPS Conference, Long Beach, CA, 9-13 February 2003, P1.10, 2003.

Trafton, J. G. and Hoffman, R. R.: Computer-aided visualization in meteorology, in: Expertise Out of Context: Proceedings of the Sixth International Conference on Naturalistic Decision Making, edited by: Hoffman, R. R., Chap. 15, Psychology Press, 337-357, 2007

Treinish, L. A.: Weather forecasting for the 1996 Olympics, IEEE Comput. Graphics Appl., 16, 10-13, 1996.

Treinish, L. A.: Task-specific visualization design: a case study in operational weather forecasting, in: Proceedings Visualization '98, Research Triangle Park, NC, 24 October 1998, 405-409, doi:10.1109/VISUAL.1998.745330, 1998.

Treinish, L. A. and Rothfusz, L. P.: Three-dimensional visualization for support of operational forecasting at the 1996 Centennial Olympic Games, in: Proceedings of the 13th IIPS Conference, 2-7 February 1997, Long Beach, CA, 2-8, 1997.

Untch, A. and Hortal, M.: A finite-element scheme for the vertical discretization of the semi-Lagrangian version of the ECMWF forecast model, Q. J. Roy. Meteor. Soc., 130, 1505-1530, 2004.

Upson, C., Faulhaber, T., Kamins, D., Laidlaw, D., Schlegel, D., Vroom, J., Gurwitz, R., and Van Dam, A.: The application visualization system: a computational environment for scientific visualization, IEEE Comput. Graphics Appl., 9, 30-42, 1989.

Vaughan, G., Methven, J., Anderson, D., Antonescu, B., Baker, L., Baker, T. P., Ballard, S. P., Bower, K. N., Brown, P. R. A., Chagnon, J., Choularton, T. W., Chylik, J., Connolly, P. J., Cook,
P. A., Cotton, R. J., Crosier, J., Dearden, C., Dorsey, J. R., Frame, T. H. A., Gallagher, M. W., Goodliff, M., Gray, S. L., Harvey, B. J., Knippertz, P., Lean, H. W., Li, D., Lloyd, G., MartínezAlvarado, O., Nicol, J., Norris, J., Öström, E., Owen, J., Parker, D. J., Plant, R. S., Renfrew, I. A., Roberts, N. M., Rosenberg, P., Rudd, A. C., Schultz, D. M., Taylor, J. P., Trzeciak, T., Tubbs, R., Vance, A. K., van Leeuwen, P. J., Wellpott, A., and Woolley, A.: Cloud Banding and Winds in Intense European Cyclones: Results from the DIAMET Project, Bull. Amer. Meteor. Soc., 96, 249-265, 2015.

Voigt, C., Schumann, U., Jurkat, T., Schäuble, D., Schlager, H., Petzold, A., Gayet, J.-F., Krämer, M., Schneider, J., Borrmann, S., Schmale, J., Jessberger, P., Hamburger, T., Lichtenstern, M., Scheibe, M., Gourbeyre, C., Meyer, J., Kübbeler, M., Frey, W., Kalesse, H., Butler, T., Lawrence, M. G., Holzäpfel, F., Arnold, F., Wendisch, M., Döpelheuer, A., Gottschaldt, K., Baumann, R., Zöger, M., Sölch, I., Rautenhaus, M., and Dörnbrack, A.: In-situ observations of young contrails - overview and selected results from the CONCERT campaign, Atmos. Chem. Phys., 10, 9039-9056, doi:10.5194/acp-10-9039-2010, 2010.

Walton, J.: NAG's IRIS Explorer, in: The Visualization Handbook, edited by Hansen, C. D. and Johnson, C., chap. 32, 633-654, Academic Press, 2005.

Wanger, L. R., Ferwerda, J. A., and Greenberg, D. P.: Perceiving spatial relationships in computer-generated images, IEEE Comput. Graph., 12, 44-58, 1992.

Watson, A. I., Fournier, J. D., Lericos, T. P., and Szoke, E. J.: The use of D3D when examining Tropical Cyclones, in: Proceedings of the 18th IIPS Conference, 13-18 January 2002, Orlando, Florida, 2002.

Watson, D.: Meteorological data visualisation using IBM Visualisation Data Explorer, in: Proceedings of the 5th ECMWF Workshop on Meteorological Operational Systems, 13-17 November 1995, Reading, England, 238-251, 1995.

Weigle, C. and Banks, D. C.: A comparison of the perceptual benefits of linear perspective and physically-based illumination for display of dense 3-D streamtubes, IEEE T. Vis. Comput. Gr., 14, 1723-1730, 2008.

Whitaker, R. T., Mirzargar, M., and Kirby, R. M.: Contour boxplots: a method for characterizing uncertainty in feature sets from simulation ensembles, IEEE T. Vis. Comput. Gr., 19, 2713-2722, 2013.

Wilhelmson, R. B., Jewett, B. F., Shaw, C., Wicker, L. J., Arrott, M., Bushell, C. B., Bajuk, M., Thingvold, J., and Yost, J. B.: A Study of the Evolution of a Numerically Modeled Severe Storm, Int. J. High Perform. C., 4, 20-36, 1990.

Wilks, D. S.: Statistical Methods in the Atmospheric Sciences, 3rd Edn., Academic Press, Amsterdam, 2011.

Wittenbrink, C. M., Pang, A. T., and Lodha, S. K.: Glyphs for visualizing uncertainty in vector fields, IEEE T. Vis. Comput. Gr., 2, 266-279, 1996.

Wulfmeyer, V., Behrendt, A., Bauer, H.-S., Kottmeier, C., Corsmeier, U., Blyth, A., Craig, G., Schumann, U., Hagen, M., Crewell, S., Di Girolamo, P., Flamant, C., Miller, M., Montani, A., Mobbs, S., Richard, E., Rotach, M. W., Arpagaus, M., Russchenberg, H., Schlüssel, P., König, M., Gärtner, V., Steinacker, R., Dorninger, M., Turner, D. D., Weckwerth, T., Hense, A., and Simmer, C.: RESEARCH CAMPAIGN: The Convective and 
Orographically Induced Precipitation Study, Bull. Amer. Meteor. Soc., 89, 1477-1486, 2008.

Yalda, S., Zoppetti, G., Clark, R., and Mackin, K.: Interactive immersion learning: flying through weather data onboard the GEOpod, B. Am. Meteorol. Soc., 93, 1811-1813, 2012.

Yessad, K.: FULL-POS in the Cycle 40T1 of ARPEGE/IFS, Tech. rep., Meteo-France, 2014.
Zehner, B., Watanabe, N., and Kolditz, O.: Visualization of gridded scalar data with uncertainty in geosciences, Comput. Geosci., 36, 1268-1275, 2010.

Zeileis, A., Hornik, K., and Murrell, P.: Escaping RGBland: selecting colors for statistical graphics, Comput. Stat. Data An., 53, 3259-3270, 2009. 